\title{
RIGIDITY PHENOMENA IN THE MAPPING CLASS GROUP
}

\author{
JAVIER ARAMAYONA \& JUAN SOUTO
}

Throughout this article we will consider connected orientable surfaces of negative Euler characteristic and of finite topological type, meaning of finite genus and with finitely many boundary components and/or cusps. We will feel free to think about cusps as marked points, punctures or topological ends. Sometimes we will need to make explicit mention of the genus and number of punctures of a surface: in this case, we will write $S_{g, n}$ for the surface of genus $g$ with $n$ punctures and empty boundary. Finally, we define the complexity of a surface $X$ as the number $\kappa(X)=3 g-3+p$, where $g$ is the genus and $p$ is the number of cusps and boundary components of $X$.

In order to avoid too cumbersome notation, we denote by

$$
\operatorname{Homeo}(X)=\left\{\begin{array}{l|l}
f: X \rightarrow X \mid \begin{array}{c}
f \text { is an orientation-preserving } \\
\text { homeomorphism fixing pointwise the } \\
\text { boundary and each puncture of } X
\end{array}
\end{array}\right\}
$$

the group of orientation-preserving self-homeomorphisms of $X$ relative to the boundary and the set of punctures. We endow $\operatorname{Homeo}(X)$ with the compactopen topology, and denote by $\mathrm{Homeo}_{0}(X)$ the connected component of the identity Id $: X \rightarrow X$. It is well-known that $\operatorname{Homeo}_{0}(X)$ consists of those elements in $\operatorname{Homeo}(X)$ that are isotopic to Id $: X \rightarrow X$ relative to $\partial X$ and the set of punctures of $X$. The mapping class group $\operatorname{Map}(X)$ of $X$ is the group

$$
\operatorname{Map}(X)=\operatorname{Homeo}(X) / \operatorname{Homeo}_{0}(X) .
$$

In the literature, $\operatorname{Map}(X)$ is sometimes referred to as the pure mapping class group. We will also need to consider the extended mapping class group $\operatorname{Map}^{*}(X)$, i.e. the group of all isotopy classes of self-homeomorphisms of $X$. Note that if $X$ has $r$ boundary components and $n$ punctures, we have an exact sequence

$$
0 \rightarrow \mathbb{Z}^{r} \rightarrow \operatorname{Map}(X) \rightarrow \operatorname{Map}^{*}(X) \rightarrow \mathbb{Z}_{2} \times \operatorname{Sym}_{r} \times \operatorname{Sym}_{n} \rightarrow 1
$$

where $\operatorname{Sym}_{s}$ is the group of permutations of the set with $s$ elements.

Let $\mathcal{T}(X)$ and $\mathcal{M}(X)=\mathcal{T}(X) / \operatorname{Map}(X)$ be, respectively, the Teichmüller and moduli spaces of $X$. The triad formed by $\operatorname{Map}(X), \mathcal{T}(X)$ and $\mathcal{M}(X)$ is often compared with the one formed, for $n \geq 3$, by $\mathrm{SL}_{n} \mathbb{Z}$, the symmetric space $\mathrm{SO}_{n} \backslash \mathrm{SL}_{n} \mathbb{R}$, and the locally symmetric space $\mathrm{SO}_{n} \backslash \mathrm{SL}_{n} \mathbb{R} / \mathrm{SL}_{n} \mathbb{Z}$.

The second author has been partially supported by NSERC Discovery and Accelerator Supplement grants. 
Here $\mathrm{SL}_{n} \mathbb{Z}$ stands as the paradigm of an arithmetic lattice in a higher rank semi-simple algebraic group. This analogy has motivated many, possibly most, advances in the understanding of the mapping class group. For example, Grossman [35] proved that $\operatorname{Map}(X)$ is residually finite; Birman, Lubotzky and McCarthy [16] proved that the Tits alternative holds for subgroups of $\operatorname{Map}(X)$; the Thurston classification of elements in $\operatorname{Map}(X)$ mimics the classification of elements in an algebraic group [91]; Harvey [37] introduced the curve complex in analogy with the Tits' building; Harer's [36] computation of the virtual cohomological dimension of $\operatorname{Map}(X)$ follows the outline of Borel and Serre's argument for arithmetic groups [18], etc... On the other hand, the comparison between $\operatorname{Map}(X)$ and $\mathrm{SL}_{n} \mathbb{Z}$ has strong limitations; for instance the mapping class group contains many infinite normal subgroups of infinite index [25], has finite index in its abstract commensurator [46], and has infinite dimensional second bounded cohomology [15]. In addition, it is not known if the mapping class group contains finite index subgroups $\Gamma$ with $H^{1}(\Gamma ; \mathbb{R}) \neq 0$. We refer to [49] for a survey on the analogy between the mapping class group and arithmetic groups.

With the dictionary between $\operatorname{Map}(X)$ and $\mathrm{SL}_{n} \mathbb{Z}$ in mind, it is natural to ask to what extent is there an analog of Margulis Superrigidity in the context of mapping class groups. There are many interpretations of this rather vague question. Our goal here is to discuss some of them, stating known results, giving here and there an argument, and proposing a few concrete questions. The plan of the paper is as follows:

In section 1 we briefly remind the reader of some rigidity theorems for irreducible lattices in higher rank semi-simple Lie groups, such as Kahzdan's theorem asserting that such lattices have property $(\mathrm{T})$, and the Mostow Rigidity and Margulis Superrigidity theorems. We highlight three flavors of the latter: a geometric version, asserting that maps between locally symmetric spaces are homotopic to totally geodesic maps; a Lie theoretic version, stating that homomorphisms between lattices arise from homomorphisms between the ambient Lie groups; and a folkloric version, asserting that the only homomorphisms between two lattices are the "obvious ones", meaning that they arise from linear algebra constructions.

In section 2 we discuss a result due to Farb-Masur [29], which states that every homomorphism from a higher rank lattice to $\operatorname{Map}(X)$ has finite image, sketching a proof due to Bridson-Wade [20]. We also discuss briefly homomorphisms from $\operatorname{Map}(X)$ to lattices, proving for example that, under any such homomorphism, Dehn twists are mapped to roots of unipotent elements. As an application, we recover a result of Bridson [19] asserting that Dehn twists are mapped to roots to multi-twists under arbitrary homomorphisms $\operatorname{Map}(X) \rightarrow \operatorname{Map}(Y)$, as long as $X$ has genus at least 3 .

This last result leads us to the main theme of this article: homomorphisms between different mapping class groups. We begin section 3 by reminding the reader of the rigidity of the curve complex and other closely related complexes. We then discuss injective endomorphisms of mapping class groups, 
and give a proof of a result due to Ivanov asserting that every automorphism of $\operatorname{Map}(X)$ is induced by a self-homeomorphism of $X$. We give this argument because it is easy and beautiful, but also because it parallels the proof of the Mostow Rigidity theorem in higher rank, replacing the Tits building by the curve complex. We end the section by giving some results that point towards the following analog of folkloric superrigidity: homomorphisms between mapping class groups arise from manipulations of surfaces.

In section 4 we consider analogs of the Lie theoretic version of superrigidity. The main problem is that, by work of Morita and Markovic, there is no ambient group for the mapping class group: $\operatorname{Map}(X)$ is a quotient of $\operatorname{Homeo}(X)$ but not a subgroup thereof. After reviewing a number of results in this direction we propose what we call the Lie theoretic version of superrigidity for homomorphisms between mapping class groups, namely that every homomorphism $\operatorname{Map}(X) \rightarrow \operatorname{Map}(Y)$ is induced by a homomorphism $\operatorname{Diff}_{c}(X) \rightarrow \operatorname{Diff}_{c}(Y)$ between the associated groups of diffeomorphisms with compact support disjoint from the boundary.

In section 5, we arrive to the geometric version of superrigidity. As will be discussed, the obvious formulation of geometric superrigidity cannot hold when we endow moduli space with any reasonable metric. However, we propose the following: Every (irreducible) homomorphism between mapping class groups induces a holomorphic map between the corresponding moduli spaces. We will finish by discussing how harmonic maps could possibly be used to prove that this is the case, and point out the main technical difficulties.

Background. We assume that the reader has some previous understanding of the mapping class group, and we refer to [28, 47] for basic facts and definitions.

This paper was written during the program "Automorphisms of Free Groups: Algorithms, Geometry and Dynamics" at the CRM, Barcelona. We would like to thank the organizers of the program, as well as to express our gratitude to the CRM for its hospitality.

\section{Classical Rigidity}

In this section we review very briefly a few rigidity results for irreducible lattices $\Gamma$ in semi-simple Lie groups $G$. We refer the reader to $[93,79]$ and to $[50,97]$ for very readable accounts of many of the topics discussed in this section. More details can be found in [11, 63, 77, 98].

We start recalling some facts and terminology on semi-simple Lie groups and lattices therein. We assume without further notice that the identity component $G_{0}$ of $G$ has finite index in $G$ - this is automatically the case if $G$ is algebraic. The universal cover $\tilde{G}_{0}$ of $G_{0}$ admits a Lie group structure so that the covering $\pi: \tilde{G}_{0} \rightarrow G_{0}$ becomes a group homomorphism. Two groups whose identity components have isomorphic universal covers are isogenous. The simply connected semi-simple group $\tilde{G}_{0}$ splits as a product 
of simple groups; these are the factors of $G$. For the sake of concreteness we assume that $G$ has no compact factors. We denote by $K$ a maximal compact subgroup of $G$ and we endow the quotient $K \backslash G$ with a $G$-invariant Riemannian metric; $K \backslash G$ is the symmetric space of non-compact type associated to $G$. Note that the metric on $K \backslash G$ is in general not unique. However, it is well-understood how these metrics relate to each other; moreover, they all have non-positive sectional curvature. The real rank of $G$,

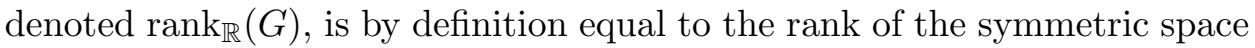
$K \backslash G$, i.e. the maximal dimension of a totally geodesic flat subspace.

A subgroup $\Gamma$ of the semi-simple group $G$ is a lattice if the associated locally symmetric space $K \backslash G / \Gamma$ has finite volume. A lattice it uniform if $K \backslash G / \Gamma$ is compact; otherwise it is non-uniform. A lattice $\Gamma \subset G$ is irreducible if the preimage of $\Gamma \cap G_{0}$ in the universal cover $\tilde{G}_{0}$ of $G_{0}$ projects densely to every factor of $\tilde{G}_{0}$; otherwise it is reducible. Notice that $\Gamma$ is reducible if and only if the locally symmetric space $K \backslash G / \Gamma$ is finitely covered by a Riemannian product.

1.1. Property $(\mathbf{T})$. Let $G$ be a compactly generated topological group, such as a Lie group or a finitely generated discrete group. A unitary action $G \curvearrowright V$ on a Hilbert space has almost invariant vectors if for every compact set $C \subset G$ and every $\epsilon>0$, there is some unit vector $v \in V$ with $\|g v-v\| \leq \epsilon$ for all $g \in C$. The group $G$ is said to have Kazhdan's property (T) if every unitary representation of $G$ that has almost invariant vectors also has some invariant unit vector. We refer to [11] for a discussion of groups with property $(\mathrm{T})$.

Perhaps the prime examples of groups with property $(\mathrm{T})$ are higher rank Lie groups and lattices therein. More precisely we have:

Theorem 1.1 (Kahzdan). A semi-simple Lie group $G$ has property $(T)$ if and only if no simple factor is isogenous to $\mathrm{SO}(1, n)$ or $\mathrm{SU}(1, n)$. Moreover, $G$ has property $(T)$ if and only if lattices therein do as well.

It follows easily from the definition given above that a group has property (T) then every finite extension and every quotient do as well. Since $\mathbb{Z}$ does not have property $(\mathrm{T})$, it follows from Kahzdan's theorem that:

Corollary 1.2. Let $\Gamma$ be a lattice in a semi-simple Lie group without factors isogenous to $\mathrm{SO}(1, n)$ or $\mathrm{SU}(1, n)$. Then $H^{1}(\Gamma ; \mathbb{R})=0$.

At this point we remind the reader of one of the deepest theorems in the theory of lattices in higher rank groups, namely the following result due to Margulis [63] asserting that normal subgroups of lattices are either finite or have finite index.

Normal Subgroup Theorem (Margulis). Let $\Gamma$ be an irreducible lattice in a semi-simple Lie group $G$ with $\operatorname{rank}_{\mathbb{R}}(G) \geq 2$. If $N \triangleleft \Gamma$ is a normal subgroup then either $N$ or $\Gamma / N$ is finite. 
Combining the Normal Subgroup Theorem and Corollary 1.2 one obtains a much more powerful version of the latter:

Corollary 1.3. Let $G$ be a semi-simple Lie group (as always without compact factors) which is not isogenous to $\mathrm{SO}(1, n)$ or $\mathrm{SU}(1, n)$. If $\Gamma \subset G$ is an irreducible lattice, then $H^{1}(\Gamma ; \mathbb{R})=0$.

1.2. Mostow Rigidity. Corollary 1.3 implies that lattices in semi-simple Lie groups non-isogenous to $\mathrm{SO}(n, 1)$ and $\mathrm{SU}(n, 1)$ do not map onto free groups, surface groups, infinite nilpotent groups, infinite solvable groups, and so on. This lack of homomorphisms can be considered as the first and most basic rigidity property of such lattices. Mostow's theorem is a rigidity result of a different kind. Namely, it asserts that isomorphisms between lattices are restrictions of automorphisms of the ambient group:

Mostow Rigidity. Let $G$ be a semi-simple Lie group without factors of dimension 3, and let $\Gamma$ and $\Gamma^{\prime}$ be irreducible lattices in $G$. Every isomorphism $\Gamma \rightarrow \Gamma^{\prime}$ is the restriction of an automorphism of $G$.

The Mostow Rigidity theorem is due to Mostow for uniform lattices $[76,77]$ and to Prasad [82] in the non-uniform case. If $\operatorname{rank}_{\mathbb{R}}(G) \geq 2$, Mostow shows that the isomorphism between the lattices $\Gamma$ and $\Gamma^{\prime}$ induces an automorphism of the Tits building associated to $G$, using then that the latter is rigid by Tits's work [92]. If $\operatorname{rank}_{\mathbb{R}}(G)=1$ a different argument is needed, and in fact there are many proofs in this case, notably for lattices in $\mathrm{SO}(n, 1)$; see for example [90]. Local rigidity, that is, when generators of $\Gamma^{\prime}$ are sufficiently close to generators of $\Gamma$, was proved first by Weil $[95,96]$ following an idea of Calabi and Vesentini for uniform lattices, and by Garland and Raghunathan in the non-uniform case [83, 34]. We refer to [26] for a discussion of the automorphisms of classical groups.

We discuss now two applications of the Mostow Rigidity theorem. Every element $A \in \mathrm{GL}_{n} \mathbb{R}$ induces the automorphism $X \mapsto A X A^{-1}$ of $\mathrm{SL}_{n} \mathbb{R}$. In fact, we obtain in this way that $\operatorname{Aut}\left(\mathrm{SL}_{n} \mathbb{R}\right)=\mathrm{PGL}_{n} \mathbb{R} \rtimes \mathbb{Z} / 2 \mathbb{Z}$ where $X \mapsto{ }^{t} X^{-1}$ is the non-trivial element $\mathbb{Z} / 2 \mathbb{Z}$. Supposing that $n \geq 3$, one gets from Mostow Rigidity that $\operatorname{Aut}\left(\mathrm{SL}_{n} \mathbb{Z}\right)$ is the subgroup of $\operatorname{Aut}\left(\mathrm{SL}_{n} \mathbb{R}\right)$ preserving $\mathrm{SL}_{n} \mathbb{Z}$ :

Corollary 1.4. Aut $\left(\mathrm{SL}_{n} \mathbb{Z}\right)=\mathrm{PGL}_{n} \mathbb{Z} \rtimes \mathbb{Z} / 2 \mathbb{Z}$ for all $n \geq 3$.

Recall that the abstract commensurator $\operatorname{Comm}(G)$ of a group $G$ is the abstract group consisting of all equivalence classes of isomorphisms between finite index subgroups of $G$, where two such isomorphisms $\phi: H_{1} \rightarrow H_{2}$ and $\phi^{\prime}: H_{1}^{\prime} \rightarrow H_{2}^{\prime}$ are equivalent if there is $H$, of finite index in both $H_{1}$ and $H_{1}^{\prime}$, such that $\left.\phi\right|_{H}=\left.\phi^{\prime}\right|_{H}$. As long as $n \geq 3$, it follows from Mostow Rigidity that $\operatorname{Comm}\left(\mathrm{SL}_{n} \mathbb{Z}\right)$ is the subgroup of $\operatorname{Aut}\left(\mathrm{SL}_{n} \mathbb{R}\right)$ consisting of those automorphisms $\phi$ such that $\mathrm{SL}_{n} \mathbb{Z} \cap \phi\left(\mathrm{SL}_{n} \mathbb{Z}\right)$ has finite index in both $\mathrm{SL}_{n} \mathbb{Z}$ and $\phi\left(\mathrm{SL}_{n} \mathbb{Z}\right)$. We get:

Corollary 1.5. $\operatorname{Comm}\left(\mathrm{SL}_{n} \mathbb{Z}\right)=\mathrm{PGL}_{n} \mathbb{Q} \rtimes \mathbb{Z} / 2 \mathbb{Z}$ for all $n \geq 3$. 
Recall that the commensurator $\operatorname{Comm}_{G}(\Gamma)$ of a subgroup $\Gamma$ of a group $G$ is the subgroup of $G$ consisting of those $g \in G$ such that $\Gamma \cap g \Gamma g^{-1}$ has finite index in both $\Gamma$ and $g \Gamma g^{-1}$. Suppose that $G$ is as in the statement of Mostow Rigidity. Then the group of inner automorphisms of $G$ has finite index in the group of all automorphisms, and hence it follows from Mostow Rigidity that the image of the obvious homomorphism

$$
\operatorname{Comm}_{G}(\Gamma) \rightarrow \operatorname{Comm}(\Gamma)
$$

has finite index. If $\Gamma$ is arithmetic, then $\Gamma$ has infinite index in $\operatorname{Comm}_{G}(\Gamma)$. A converse to this fact is due to Margulis [63]:

Theorem 1.6 (Margulis). Let $G$ be a semi-simple Lie group and $\Gamma \subset G$ an irreducible lattice. Then $\Gamma$ is arithmetic if and only if $\Gamma$ has infinite index in $\operatorname{Comm}_{G}(\Gamma)$.

1.3. Superrigidity. While Mostow Rigidity is concerned on the rigidity of isomorphisms between lattices of the same ambient Lie group, Margulis Superrigidity theorem is a rigidity theorem for arbitrary homomorphisms from lattices in a Lie group to another Lie group [63, 98]:

Margulis Superrigidity. Let $G$ and $G^{\prime}$ be connected Lie groups with trivial center and no compact factors. Suppose that $\operatorname{rank}_{\mathbb{R}}(G) \geq 2$ and let $\Gamma \subset G$ be an irreducible lattice. Then, every homomorphism $\phi: \Gamma \rightarrow G^{\prime}$ with Zariski dense image extends to a homomorphism $\hat{\phi}: G \rightarrow G^{\prime}$.

We have chosen a rather restrictive version of the Margulis Superrigidity theorem, assuming for instance that $\phi$ has Zariski dense image. The more general statement, which follows easily from the formulation above, remains the same "up to compact groups". For instance, for non-uniform lattices in $\mathrm{SL}_{k} \mathbb{R}$ we have:

Corollary 1.7. Suppose that $k \geq 3$, that $\Gamma \subset \mathrm{SL}_{k} \mathbb{R}$ is a non-uniform lattice and that $\phi: \Gamma \rightarrow \mathrm{GL}_{n} \mathbb{R}$ is a homomorphism. Then there is a homomorphisms $\Phi: \mathrm{SL}_{k} \mathbb{R} \rightarrow \mathrm{GL}_{n} \mathbb{R}$ whose restriction to a finite index subgroup of $\Gamma$ agrees with $\phi$.

A homomorphism $\mathrm{SL}_{k} \mathbb{R} \rightarrow \mathrm{GL}_{n} \mathbb{R}$ is, by definition, the same as an $n$ dimensional representation of $\mathrm{SL}_{k} \mathbb{R}$. All these are, or at least can in principle be, classified. In particular, in extremely vague and colloquial language, one can interpret Margulis's theorem as follows:

Folkloric version of superrigidity. Let $G$ and $G^{\prime}$ be semi-simple Lie groups, with $\operatorname{rank}_{\mathbb{R}}(G) \geq 2$, and $\Gamma \subset G$ a lattice. Every homomorphism $\Gamma \rightarrow G^{\prime}$ is one of the "obvious" ones.

Margulis Superrigidity has also a geometric formulation. Suppose that $G, G^{\prime}$ are semi-simple Lie groups and $K \subset G$ and $K^{\prime} \subset G^{\prime}$ maximal compact 
subgroups. Denote by $S=K \backslash G$ and $S^{\prime}=K^{\prime} \backslash G^{\prime}$ be the associated symmetric spaces, endowed respectively with $G$-invariant and $G^{\prime}$-invariant Riemannian metrics. Recall that both $S$ and $S^{\prime}$ are simply connected complete manifolds with non-positive sectional curvature, and hence contractible. In particular, if $\Gamma \subset G$ and $\Gamma^{\prime} \subset G^{\prime}$ are, say for simplicity torsion-free, lattices, then there is a one-to-one correspondence between the sets of conjugacy classes of homomorphisms $\Gamma \rightarrow \Gamma^{\prime}$ and of free homotopy classes of maps $S / \Gamma \rightarrow S / \Gamma^{\prime}$. In these terms, Margulis's theorem implies that in every homotopy class of maps there is a totally geodesic map, i.e. one which maps geodesics to geodesics:

Geometric Superrigidity (Margulis). Let $M$ and $M^{\prime}$ be irreducible locally symmetric spaces of finite volume. If $\operatorname{rank}_{\mathbb{R}}(M) \geq 2$, then every map $M \rightarrow$ $M^{\prime}$ is homotopic to a totally geodesic map.

This version of Geometric Superrigidity is very restrictive; a more general version is in fact equivalent to the Margulis Superrigidity theorem itself. Note also that there is no assumption on the rank of $M^{\prime}$. In fact, there are versions of the Geometric Superrigidity theorem allowing for the target to be just a manifold of non-positive curvature operator (see for example [51]).

\section{Homomorphisms Between Lattices And $\operatorname{Map}(X)$}

When trying to extend superrigidity results to the setting of mapping class groups, it is natural to suppose first that one of the involved groups is a lattice in a higher rank Lie group. As we will discuss below, while the situation for homomorphisms from lattices to mapping class groups is completely settled, we are very far from understanding the possible homomorphisms from mapping class groups to linear groups.

2.1. From lattices to $\operatorname{Map}(X)$. On the one hand, lattices are residually finite. On the other, every finite group is a subgroup of some mapping class group. Combining these two facts we obtain many homomorphisms from lattices to mapping class groups, all of them with finite image. The content of the following theorem, proved by Farb-Masur [29] building on earlier results by Ivanov and Kaimanovich-Masur [52], is that there are no other homomorphisms from lattices to mapping class groups.

Theorem 2.1 (Farb-Masur). Let $\Gamma$ be an irreducible lattice in semi-simple Lie group $G$ with $\operatorname{rank}_{\mathbb{R}}(G) \geq 2$. Then any homomorphism $\Gamma \rightarrow \operatorname{Map}(X)$ has finite image.

In the case when $X=S_{0, n}$ is a punctured sphere, this theorem follows easily from Corollary 1.3 and an induction argument. Indeed, noting that $\operatorname{Map}\left(S_{0,3}\right)$ is trivial, we may suppose that every homomorphism from a lattice to $\operatorname{Map}\left(S_{0, n-1}\right)$ is trivial. Filling in a puncture of $S_{0, n}$ we obtain a homomorphism $\operatorname{Homeo}\left(S_{0, n}\right) \rightarrow \operatorname{Homeo}\left(S_{0, n-1}\right)$ which induces a homomorphism at the level of mapping class groups. In fact, we have the following 
version of the Birman exact sequence:

$$
1 \rightarrow \pi_{1}\left(S_{0, n-1}\right) \rightarrow \operatorname{Map}\left(S_{0, n}\right) \rightarrow \operatorname{Map}\left(S_{0, n-1}\right) \rightarrow 1
$$

Our induction hypothesis implies that the image of $\Gamma \rightarrow \operatorname{Map}\left(S_{0, n}\right)$ is contained in $\pi_{1}\left(S_{0, n-1}\right)$, a free group. Corollary 1.3 then yields that every homomorphism $\Gamma$ to a free group is trivial. This proves the Farb-Masur theorem if $X$ is a punctured sphere.

The argument we just gave can also be used if $X$ has genus at most 2, but for the general case, deeper properties of the mapping class group are necessary. For instance, prior to the work of Kaimanovich-Masur and FarbMasur, Ivanov had already proved Theorem 2.1 for non-uniform lattices using the fact that solvable subgroups of $\operatorname{Map}(X)$ are virtually abelian [16]. A completely independent proof for uniform lattices is due to BestvinaFujiwara [15]. Namely, they proved that any subgroup $\Lambda$ of $\operatorname{Map}(X)$ that is not virtually abelian has infinitely generated second bounded cohomology $H_{b}^{2}(\Lambda ; \mathbb{R})$. On the other hand, Burger-Monod [21] proved that if $\Gamma$ is a uniform irreducible lattice in a higher rank group then the homomorphism $H_{b}^{2}(\Gamma ; \mathbb{R}) \rightarrow H^{2}(\Gamma ; \mathbb{R})$ is injective and hence the former is finitely generated. This implies that any homomorphism $\Gamma \rightarrow \operatorname{Map}(X)$ has virtually abelian image and now one concludes using again Corollary 1.3.

To finish the discussion of Theorem 2.1, we sketch an alternative beautiful argument due to Bridson-Wade [20]. Suppose that $\phi: \Gamma \rightarrow \operatorname{Map}(X)$ is a homomorphism with infinite image. From Corollary 1.3 we obtain that its image is not virtually abelian and hence cannot consist solely of roots of multi-twists. This implies that we can assume, up to replacing $\Gamma$ by a finite index subgroup and $X$ by an open subsurface, that the image $\phi(\Gamma) \subset$ $\operatorname{Map}(X)$ of $\phi$ contains a pseudo-Anosov element $f$. It is known - see [25] for much more powerful results - that there is $n$ such that the normal closure $H=\left\langle\left\langle f^{n}\right\rangle\right\rangle$ of the $n$-th power of $f$ is an infinitely generated free subgroup of $\operatorname{Map}(X)$. It follows that $\phi^{-1}(H)$ is an infinite normal subgroup of $\Gamma$. From the Normal Subgroup Theorem we derive that $\phi^{-1}(H)$ is itself a lattice. By construction $\phi^{-1}(H)$ maps non-trivially to the free group $H$, contradicting Corollary 1.3. This proves the Farb-Masur Theorem.

2.2. From $\operatorname{Map}(X)$ to lattices. While Theorem 2.1 asserts that there are no interesting homomorphisms from lattices to mapping class groups, there are many homomorphisms in the other direction. For instance, suppose that $X$ is surface of genus $g$. The action of $\operatorname{Map}(X)$ on the first integer homology of $X$ induces an action $\operatorname{Map}(X) \curvearrowright \mathbb{Z}^{2 g}$ : if $X$ is closed then $\mathbb{Z}^{2 g} \simeq H_{1}(X ; \mathbb{Z})$; otherwise $\mathbb{Z}^{2 g}$ is the quotient of $H_{1}(X ; \mathbb{Z})$ by the submodule generated by cycles parallel to the boundary or which bound a punctured disk in $X$. The action $\operatorname{Map}(X) \curvearrowright \mathbb{Z}^{2 g}$ preserves the unimodular non-degenerate alternating bilinear form induced by the algebraic intersection number. In other words we obtain the symplectic representation

$$
\operatorname{Map}(X) \rightarrow \operatorname{Sp}_{2 g}(\mathbb{Z})
$$


This homomorphism is surjective, but very far from injective. Its kernel, the Torelli group $\operatorname{Tor}(X)$, is an infinite group as long as $g \geq 2$. In fact, Mess [74] proved that the Torelli group of a closed surface of genus 2 is an infinitely generated free group.

Suppose now that $\pi: X^{\prime} \rightarrow X$ is a finite cover. There is a finite index subgroup $\mathcal{G}$ of $\operatorname{Homeo}(X)$ that lifts to $\operatorname{Homeo}\left(X^{\prime}\right)$, meaning that there is a continuous homomorphism

$$
\mathcal{G} \rightarrow \operatorname{Homeo}\left(X^{\prime}\right), \quad f \mapsto \hat{f}
$$

so that $\pi(\hat{f}(x))=f(\pi(x))$ for all $x \in X^{\prime}$. This homomorphism induces a homomorphism $\Gamma \rightarrow \operatorname{Map}\left(X^{\prime}\right)$ where $\Gamma$ is the finite index $\operatorname{subgroup}$ of $\operatorname{Map}(X)$ whose elements are represented by elements in $\mathcal{G}$. Now, composing this homomorphism with the symplectic representation $\operatorname{Map}\left(X^{\prime}\right) \rightarrow \operatorname{Sp}_{2 g^{\prime}}(\mathbb{Z})$, where $g^{\prime}$ is the genus of $X^{\prime}$, we obtain a representation

$$
\Gamma \rightarrow \mathrm{Sp}_{2 g^{\prime}}(\mathbb{Z})
$$

The representations (2.1) have been studied by Looijenga [59] for abelian covers $\pi: X^{\prime} \rightarrow X$, who used them to prove that there are epimorphisms of finite index subgroups of $\operatorname{Map}(X)$ onto arithmetic groups with arbitrarily large $\mathbb{Q}$-rank.

Remark. Notice that since $\Gamma$ has finite index in $\operatorname{Map}(X)$ we can induce up this representation and obtain a homomorphism $\operatorname{Map}(X) \rightarrow \operatorname{Sp}_{2 g^{\prime \prime}}(\mathbb{Z})$ for some suitable choice of $g^{\prime \prime}$.

Although there is no general result in this direction, it is to be expected that none of the representations (2.1) are faithful. On the other hand, Koberda [55] proved that for every non-trivial $f \in \operatorname{Map}(X)$ there is a finite cover $\pi: X^{\prime} \rightarrow X$ such that $f$ belongs to the domain of (2.1) but not to the kernel.

Continuing with the same notation, note that the representation $(2.1)$ is not irreducible: the kernel of $H_{1}(\pi): H_{1}\left(X^{\prime} ; \mathbb{Z}\right) \rightarrow H_{1}(X, \mathbb{Z})$ is an invariant subspace. Suppose that $X$ has genus 2 and $X^{\prime}$ genus 3 ; hence $\pi$ has degree 2. Restricting $(2.1)$ to $\operatorname{Ker}\left(H_{1}(\pi)\right)$ we obtain a finite index subgroup $\Gamma \subset$ $\operatorname{Map}(X)$ and a representation

$$
\Gamma \rightarrow \mathrm{SL}_{2} \mathbb{Z}=\operatorname{Aut}\left(\operatorname{Ker}\left(H_{1}(\pi)\right)\right)
$$

whose image has finite index. Noting that $\mathrm{SL}_{2} \mathbb{Z}$ is virtually free we get hence that $\operatorname{Map}(X)$ virtually surjects onto a free group. We give now a different construction, more aligned with the spirit of this paper, for such homomorphisms:

Lemma 2.2. If $X$ has genus at most 2 , then there is a finite index subgroup of $\operatorname{Map}(X)$ that surjects onto a non-abelian free group.

Proof. We prove the claim only if $X$ has genus 2, leaving the other cases to the reader. To begin with, let $\bar{X}$ be the surface obtained from $X$ by filling in all punctures and capping off all boundary components with disks. The 
embedding of $X$ into $\bar{X}$ induces a homomorphism $\operatorname{Homeo}(X) \rightarrow \operatorname{Homeo}(\bar{X})$; in fact the induced homomorphism $\operatorname{Map}(X) \rightarrow \operatorname{Map}(\bar{X})$ is surjective. It hence follows that it suffices to prove the claim for $\bar{X}$.

The hyperelliptic involution $\tau$ of $\bar{X}$ is central in $\operatorname{Map}(\bar{X})$ and every element in $\operatorname{Map}(\bar{X})$ is represented by a $\tau$-equivariant homeomorphism. Thus, there is a sujective homomorphism

$$
\pi: \operatorname{Map}(X) \rightarrow \operatorname{Map}^{*}(X / \tau)
$$

to the extended mapping class group of the 6-punctured sphere $X / \tau=$ $S_{0,6}$; here we understand the orbifold points of $X / \tau$ as marked points. The mapping class group $\operatorname{Map}(X / \tau)$ has finite index in the extended mapping class group $\operatorname{Map}^{*}(X / \tau)$ and surjects onto $\operatorname{Map}\left(S_{0,4}\right)$, a free group (compare with the paragraph after the statement of Theorem 2.1). Hence the claim follows.

Since groups with property $(\mathrm{T})$ do not virtually surject onto free groups we get:

Corollary 2.3. Suppose that $X$ is a surface of finite topological type and genus $g \leq 2$. Then $\operatorname{Map}(X)$ does not have property $(T)$.

Andersen [2] has announced that mapping class groups of arbitrary surfaces of finite topological type also fail to have property $(\mathrm{T})$. This does however not say anything about the existence of homomorphisms from finite index subgroups of $\operatorname{Map}(X)$ onto $\mathbb{Z}$, or even onto non-abelian free groups. This is one of the outstanding open problems in this field:

Question 1. Do mapping class groups of surfaces of genus $g \geq 3$ virtually surject onto free groups?

Clearly, if the answer to Question 1 is positive, then it is impossible to classify all homomorphisms from $\operatorname{Map}(X)$ to lattices. On the other hand, it is possible to prove that there are no non-trivial representations $\operatorname{Map}(X) \rightarrow \mathrm{GL}_{n} \mathbb{C}$ if $n$ is relatively small with respect to the genus of $X$. For instance, Franks-Handel [33] showed that every homomorphism $\operatorname{Map}\left(S_{g, 0}\right) \rightarrow \mathrm{GL}_{n} \mathbb{C}$ is trivial if $n<2 g$. In this spirit we wish to point out that Kielak [54] has proved that, as long as $n \geq 6$ and $m<\frac{n(n+1)}{2}$, every homomorphism $\operatorname{Out}\left(\mathbb{F}_{n}\right) \rightarrow \mathrm{GL}_{m} \mathbb{Z}$ factors through the natural projection $\operatorname{Out}\left(\mathbb{F}_{n}\right) \rightarrow \mathrm{GL}_{n} \mathbb{Z}$. A similar result should hold for the mapping class group as well. However, Kielak uses extensively that Out $\left(\mathbb{Z}_{n}\right)$ contains rather large finite groups such as $\mathbb{Z}_{2}^{n} \rtimes \mathrm{Sym}_{n}$ or $\mathbb{Z}_{2} \times \mathrm{Sym}_{n+1}$ - on the other hand, the mapping class group of a surface of genus $g$ has no finite subgroups with more than $84(g-1)$ elements. Nevertheless, the following very useful observation might come handy when trying to prove a $\operatorname{Map}(X)$ analog of Kielak's theorem:

Proposition 2.4. Suppose that $X$ is a surface of genus $g \geq 3$ and $\Gamma \subset$ $\operatorname{Map}(X)$ a finite index subgroup. For every $n \in \mathbb{N}$, there is $k \in \mathbb{N}$ such that 
for every homomorphism $\rho: \Gamma \rightarrow \mathrm{GL}_{n} \mathbb{C}$ and every multi-twist $\mu \in \Gamma$, we have that $\rho(\mu)^{k}$ is unipotent.

Proposition 2.4 will follow easily once we have proved the following:

Lemma 2.5. Suppose that $X$ is a surface of genus $g \geq 2, \delta_{\gamma} \in \operatorname{Map}(X)$ a Dehn twist along a boundary component $\gamma$ of $X, \Gamma \subset \operatorname{Map}(X)$ a finite index subgroup, and $m$ such that $\delta_{\gamma}^{m} \in \Gamma$. For every $n \in \mathbb{N}$ there is $k \in \mathbb{N}$ such that for every homomorphism $\rho: \Gamma \rightarrow \mathrm{GL}_{n} \mathbb{C}$ we have that $\rho\left(\delta_{\gamma}^{m}\right)^{k}$ is unipotent.

Proof. Let $d$ be the index of $\Gamma$ in $\operatorname{Map}(X)$, and note that we can induce up the representation $\rho$ to a representation $\operatorname{Map}(X) \rightarrow \mathrm{GL}_{n d} \mathbb{C}$ whose restriction to $\Gamma$ is the sum of $d$ copies of $\rho$. In other words, we can assume that $\rho$ was defined on $\operatorname{Map}(X)$ to begin with, and so $m=1$.

Let $\lambda_{1}, \ldots, \lambda_{r}$ be the eigenvalues of the linear transformation $\rho\left(\delta_{\gamma}\right)$ and consider the decomposition of $\mathbb{C}^{n}$ into generalized eigenspaces

$$
\mathbb{C}^{n}=E\left(\lambda_{1}\right) \oplus \cdots \oplus E\left(\lambda_{r}\right) .
$$

Note that the spaces $E\left(\lambda_{i}\right)$ are $\rho(\operatorname{Map}(X))$-invariant because $\delta_{\gamma}$ is central in $\operatorname{Map}(X)$. In particular, the homomorphism

$$
\operatorname{Map}(X) \rightarrow \mathbb{C}^{*}, \quad \phi \mapsto \operatorname{det}\left(\left.\rho(\phi)\right|_{E_{\lambda_{i}}}\right)
$$

is well-defined for $i=1, \ldots, r$. On the other hand, this homomorphism has finite image because $\mathbb{C}^{*}$ is abelian and $H^{1}(\operatorname{Map}(X) ; \mathbb{R})=0$ [57]. This implies that $\lambda_{i}$ is a root of unity for all $i$, and thus the claim follows.

We are now ready to prove Proposition 2.4:

Proof of Proposition 2.4. First, note that it suffices to prove the claim if $\mu=\delta_{\gamma}^{m}$ is a power of a Dehn twist along a single curve $\gamma$. Assuming that this is the case, let $X_{\gamma}$ be the complement of an open regular neighborhood of $\gamma$ in $X$, and observe that a connected component $Z$ of $X_{\gamma}$ has genus at least 2. The embedding $\iota: Z \rightarrow X$ induces a continuous homomorphism $\operatorname{Homeo}(Z) \rightarrow \operatorname{Homeo}(X)$, and hence a homomorphism

$$
\iota_{\#}: \operatorname{Map}(Z) \rightarrow \operatorname{Map}(X) .
$$

Moreover, there is a boundary component $\eta$ of $Z$ such that $\iota_{\#}\left(\delta_{\eta}\right)=\delta_{\gamma}$. From Lemma 2.5 we obtain that $\rho\left(\delta_{\gamma}^{m}\right)=\left(\rho \circ \iota_{\#}\right)\left(\delta_{\eta}^{m}\right)$ is a root of a unipotent element, as we needed to prove.

Observing that a compact Lie group $G$ has no unipotent elements other than the identity we deduce:

Corollary 2.6. Suppose that $X$ has genus $\geq 3, \Gamma \subset \operatorname{Map}(X)$ a finite index subgroup and $G$ a compact Lie group. There is $k$ such that for every homomorphism $\rho: \Gamma \rightarrow G$ we have $\rho(\mu)^{k} \in \operatorname{Ker}(\rho)$, for every multi-twist $\mu \in \Gamma$. 
Notice that there are many representations with infinite image of $\operatorname{Map}(X)$ into compact Lie groups. A source of such examples are the so-called quantum representations; see [66] for a discussion of this topic.

The argument used in the proof of Proposition 2.4 also imposes restrictions on the image of Dehn twists under homomorphisms to groups which a priori are not linear. For example, we recover the following result due to Bridson [19]:

Theorem 2.7 (Bridson). Suppose that $X, Y$ are surfaces of finite type, $X$ of genus at least 3 , and let $\Gamma \subset \operatorname{Map}(X)$ be a finite index subgroup. Any homomorphism $\phi: \Gamma \rightarrow \operatorname{Map}(Y)$ maps multi-twists to roots of multi-twists.

Proof. As was the case in the proof of Proposition 2.4, it suffices to prove the claim for powers of Dehn twists $\delta_{\gamma}^{m} \in \Gamma$. We also assume, for the sake of concreteness, that $\gamma$ is non-separating and hence that the complement $X_{\gamma}$ of an open regular neighborhood of $\gamma$ in $X$ is connected and has genus at least 2 . Let

$$
\iota_{\#}: \operatorname{Map}\left(X_{\gamma}\right) \rightarrow \operatorname{Map}(X)
$$

be the homomorphism induced by the inclusion of $X_{\gamma}$ in $X$, and let $\eta$ be a boundary component of $X_{\gamma}$ with $\iota_{\#}\left(\delta_{\eta}\right)=\delta_{\gamma}$.

Seeking a contradiction, suppose that $\phi\left(\delta_{\gamma}^{m}\right)$ is not a root of a multitwist. In other words, there is a $\phi\left(\delta_{\gamma}^{m}\right)$-invariant open $\pi_{1}$-injective subsurface $Z \subset Y$ of negative Euler characteristic on which $\phi\left(\delta_{\gamma}^{m}\right)$ acts as a pseudo-Anosov element. Suppose that $Z$ is the, up to isotopy, largest such subsurface, let $\lambda \subset Z$ be the recurrent geodesic lamination supporting all attracting laminations of $\delta_{\gamma}^{m}$, and let $M_{\lambda}$ be the space of measured laminations supported by $\lambda$. Note that $M_{\lambda}$ is an open convex set in a finite dimensional real vector space $V_{\lambda}$. The centralizer $\mathcal{Z}_{\operatorname{Map}(Y)}\left(\phi\left(\delta_{\gamma}^{m}\right)\right)$ of $\phi\left(\delta_{\gamma}^{m}\right)$ in $\operatorname{Map}(Y)$ preserves $Z$ and $\lambda$, and acts by linear transformations on $V_{\lambda}$, meaning that we have a homomorphism

$$
\rho: \mathcal{Z}_{\operatorname{Map}(Y)}\left(\phi\left(\delta_{\gamma}^{m}\right)\right) \rightarrow \mathrm{GL}\left(V_{\lambda}\right)
$$

By construction, the image of $\phi\left(\delta_{\gamma}^{m}\right)=\left(\phi \circ \iota_{\#}\right)\left(\delta_{\eta}^{m}\right)$ is semi-simple of infinite order. In other words, the homomorphism $\phi \circ \iota_{\#}$ contradicts Lemma 2.5.

Bridson's original proof [19] of Theorem 2.7 made use of the action of $\operatorname{Map}(Y)$ on the Weil-Peterson completion of Teichmüller space, which is a CAT(0) space. In those terms, Lemma 2.5 amounts to saying that central elements in groups $\Gamma$ with $H^{1}(\Gamma ; \mathbb{R})=0$ do not act as infinite order semisimple isometries of $\mathrm{CAT}(0)$ spaces. In fact, Lemma 2.5 follows from this assertion. However, the proof of Theorem 2.7 we present here has the virtue that it applies to other situations where no CAT(0) geometry is available: for instance, the same argument applies, once we replace laminations by trees, to prove that if $X$ has at least genus 3 , then every homomorphism $\Gamma \rightarrow \operatorname{Out}\left(\mathbb{F}_{n}\right)$ from a finite index subgroup $\Gamma \subset \operatorname{Map}(X)$ to the group of outer 
automorphisms of a free group maps multi-twists to polynomially growing automorphisms.

To conclude this section, recall that Thurston's classification of the elements in the mapping class group mimics the classification of elements in Lie groups as unipotents, semi-simple, or of mixed type. In this analogy, multi-twists are the analogue of unipotents, and pseudo-Anosov of semisimple elements. Homomorphisms between Lie groups preserve the Jordan decomposition and hence the type. Seen in this light, Proposition 2.4 and Theorem 2.7 assert that the "type" of multi-twists is preserved. On the other hand it is well-known that, in general, type is not preserved. For instance, Papadopoulos [80] noted that every element in $\operatorname{Sp}_{2 g}(\mathbb{Z})$ is the image of a pseudo-Anosov element under the symplectic representation. As we will see below (Theorem 3.10) there are also homomorphisms between mapping class groups mapping pseudo-Anosov elements to multi-twists.

\section{Combinatorial SUPERrigidity FOR MAPPING ClASS GROUPS}

As mentioned earlier, the folkloric version of Mostow and Margulis superrigidity asserts that the only homomorphisms between lattices are the "obvious ones". In light of this, one may wonder whether there is an analogous phenomenon in the context of mapping class groups, namely if every homomorphism $\operatorname{Map}(X) \rightarrow \operatorname{Map}(Y)$, at least subject to suitable conditions on $X$ and $Y$, is induced by a manipulation of the underlying surfaces. A natural starting point for the problem is to study automorphisms of the mapping class group, or isomorphisms between finite index subgroups thereof. Such isomorphisms are induced by a self-homeomorphism of $X$, by work of Ivanov. The key tool to prove this result, by itself analogous to Mostow Rigidity, is to use the rigidity of the curve complex or of one of the other similar complexes built from multicurves on $X$. We start reviewing the rigidity of such complexes (see also [72]), then discuss automorphisms and injective endomorphisms of $\operatorname{Map}(X)$, and conclude this section by reviewing what is known about homomorphisms between different mapping class groups.

3.1. Simplicial rigidity. Let $X$ be a surface of finite topological type. The curve complex $\mathcal{C}(X)$ is the simplicial complex whose vertices are homotopy classes of essential simple closed curves on $X$, and where a set of vertices of $\mathcal{C}(X)$ spans a simplex if the corresponding curves have representatives that are pairwise distinct and disjoint. Observe that $\operatorname{Map}^{*}(X)$ acts on $\mathcal{C}(X)$ by simplicial automorphisms.

The curve complex was introduced by Harvey [37] in analogy with Tits buildings for Lie groups, and has since been used to prove a number of results that highlight the comparison between mapping class groups and lattices in Lie groups. For instance, Borel and Serre [18] used the rational Tits building associated to an arithmetic group to compute the virtual cohomological dimension of the group. Similar arguments using the curve complex allowed Harer [36] to calculate the virtual cohomological dimension 
of $\operatorname{Map}(X)$. Crucially, the curve complex encodes the structure of the thin part of Teichmüller space in a similar fashion as a rational Tits building reflects the combinatorics of the end of the locally symmetric space associated to an arithmetic group.

Tits [92] proved that Tits buildings are simplicially rigid; more concretely, if $B$ is an irreducible thick spherical building of rank at least 2 associated to a linear algebraic group $G$, then every simplicial automorphism of $B$ is induced by an automorphism of $G$. The corresponding result for curve complexes is originally due to Ivanov [45], and was later extended by Korkmaz [56] and Luo [60]:

Theorem 3.1 (Ivanov). If $X$ has complexity $\kappa(X) \geq 2$ and $X \neq S_{1,2}$, then every automorphism $\mathcal{C}(X) \rightarrow \mathcal{C}(X)$ is induced by a homeomorphism $X \rightarrow X$.

Recall that the complexity of a surface $X$ is defined as the number $\kappa(X)=$ $3 g-3+p$, where $g$ and $p$ are, respectively, the genus and the number of punctures and boundary components of $X$. If $\kappa(X)=1$, then the curve complex $\mathcal{C}(X)$ is an infinite discrete set and, as such, has all sorts of automorphisms. The exceptional case of $S_{1,2}$ is somewhat different. Indeed, Luo [60] observed that the natural two-fold branched cover $S_{1,2} \rightarrow S_{0,5}$ induces an isomorphism $\mathcal{C}\left(S_{1,2}\right) \simeq \mathcal{C}\left(S_{0,5}\right)$ between curve complexes. Moreover, $\operatorname{Map}^{*}\left(S_{0,5}\right)$ acts transitively on $\mathcal{C}\left(S_{0,5}\right)$. It hence follows that there is an automorphism of $\mathcal{C}\left(S_{1,2}\right)$ which takes a separating curve to a non-separating one, and thus cannot be induced by a homeomorphism of $S_{1,2}$. On the other hand, Luo [60] proved that every automorphism of $\mathcal{C}\left(S_{1,2}\right)$ mapping non-separating curves to non-separating curves is induced by a surface homeomorphism.

As it turns out, it is possible to relax the condition of the self-map of the curve complex being an automorphism. In this direction, combining results of Irmak [41, 42], Bell-Margalit [12] and Behrstock-Margalit [10], one obtains the analog of Theorem 3.1 for superinjective self-maps of the curve complex; here, a map $\mathcal{C}(X) \rightarrow \mathcal{C}(X)$ is superinjective if it maps pairs of curves that intersect to pairs of curves that intersect. More generally, Shackleton [86] proved that the conclusion of Theorem 3.1 remains valid for locally injective simplicial maps $\mathcal{C}(X) \rightarrow \mathcal{C}(X)$, namely those which are injective on the star of every vertex of $\mathcal{C}(X)$ :

Theorem 3.2 (Shackleton). If $X$ has complexity $\kappa(X) \geq 2$ and $X \neq S_{1,2}$, then every locally injective simplicial map $\mathcal{C}(X) \rightarrow \mathcal{C}(X)$ is induced by a homeomorphism $X \rightarrow X$.

Besides the curve complex, there are various other $\operatorname{Map}(X)$-invariant "multicurve complexes" built from simple closed curves on a surface. Some of these are subcomplexes of $\mathcal{C}(X)$ spanned by a given class of vertices of $\mathcal{C}(X)$. For example, the non-separating curve complex $\mathcal{C}_{n s}(X)$ is the subcomplex of $\mathcal{C}(X)$ whose vertices correspond to non-separating curves. 
Other such complexes have as vertices a given type of multicurve on the surface, and adjacency corresponds to performing "elementary moves" on that type of multicurve. A notable example is the pants complex $\mathcal{P}(X)$, whose vertices are homotopy classes of pants decompositions of $X$, and where two pants decompositions are adjacent in $\mathcal{P}(X)$ if they share all but one curves, and the remaining two curves either fill a 4-holed sphere and intersect exactly twice, or they fill a 1-holed torus and intersect exactly once. Another example, somewhere intermediate between $\mathcal{C}(X)$ and $\mathcal{P}(X)$, is the Hatcher-Thurston complex $\mathcal{H} \mathcal{T}(X)$. The vertices of $\mathcal{H} \mathcal{T}(X)$ correspond to cut systems of $X$, namely sets of $g$ curves that together do not separate $X$, and two vertices are adjacent if they share $g-1$ curves, and the remaining two curves intersect exactly once.

Again in analogy with the simplicial rigidity of buildings, a common theme has been to prove that any automorphism of any such complex is induced by a homeomorphism of the underlying surface. This was done by Irmak [43] for the non-separating curve complex, by Margalit [62] for the pants complex, and by Irmak-Korkmaz [44] for the Hatcher-Thurston complex. In every single case, the proof boils down to showing that an automorphism of the complex in question induces an automorphism of the curve complex, and then applying Theorem 3.1. We refer to McCarthy-Papadopoulos [72] for an overview of these and various other related results.

It is in fact reasonable to expect that, for any complex of multicurves $\mathcal{K}(X)$ associated to $X$ for which there is no obvious obstruction such as being disconnected, every automorphism (resp. locally injective, injective or superinjective) simplicial map $\mathcal{K}(X) \rightarrow \mathcal{K}(X)$ is induced by a homeomorphism $X \rightarrow X$.

A more challenging problem is to understand all possible injections between complexes of multicurves associated to two different surfaces:

Question 2. Let $\mathcal{K}(X)$ and $\mathcal{K}(Y)$ be complexes of multicurves associated to distinct surfaces $X$ and $Y$. Understand all (locally) injective, or superinjective, simplicial maps $\mathcal{K}(X) \rightarrow \mathcal{K}(Y)$.

In most cases, obvious examples of simplicial injections $\mathcal{K}(X) \rightarrow \mathcal{K}(Y)$ are given by subsurface inclusions $\iota: X \rightarrow Y$. Indeed, for curve complexes, a subsurface inclusion $\iota: X \rightarrow Y$ induces an injective simplicial map $\psi$ : $\mathcal{C}(X) \rightarrow \mathcal{C}(Y)$ by the rule $\psi(\alpha)=\iota(\alpha)$; in the case of the pants complex, $\iota$ induces an injective simplicial map $\psi: \mathcal{P}(X) \rightarrow \mathcal{P}(Y)$ by first choosing a multicurve $Q \subset Y$ which is the union of $\partial(\iota(X))$ and a pants decomposition of $Y \backslash \iota(X)$, and then setting $\psi(P)=\iota(P) \cup Q$, for every $P \in \mathcal{P}(X)$.

A more bewildering construction of injective maps between curve complexes is the following. Let $X$ be a surface with boundary, and let $\iota: X \rightarrow Y$ be a subsurface inclusion such that $Y \backslash X$ is not a pair of pants. Choose curves $\alpha \subset X$ and $\beta \subset Y \backslash X$. Then the simplicial map $\phi: \mathcal{C}(X) \rightarrow \mathcal{C}(Y)$ given by $\phi(\gamma)=\iota(\gamma)$ for all $\gamma \neq \alpha$, and $\phi(\alpha)=\beta$, is injective (but not superinjective). 
Another class of examples of injections between curve complexes comes from "puncturing" a surface. To do so endow $X$ with a hyperbolic structure and choose a point $x \in X$ in the complement of the union of all simple closed geodesics on $X$; this can be done because there are only countably many such geodesics. In this way we obtain a natural injective simplicial map $\mathcal{C}(X) \rightarrow \mathcal{C}(X \backslash x)$. Moreover, using for example a result of Birman-Series [17], we may choose two discs in the complement of the union of all simple closed geodesics on $X$. Denoting by $Y$ the surface obtained from $X$ by removing the interior of each disc, and then gluing together the boundaries of the discs, we obtain an injection $\mathcal{C}(X) \rightarrow \mathcal{C}(Y)$; observe that genus $(Y)=\operatorname{genus}(X)+1$.

As a general principle, the rigidity of complexes of multicurves seems to increase with the cardinality of the multicurve representing a vertex. The following result, proved in [3], is an extreme case of this behavior:

Theorem 3.3 (Aramayona [3]). Let $X$ and $Y$ be compact orientable surfaces and assume that $\kappa(X) \geq 2$. Every locally injective simplicial map $\mathcal{P}(X) \rightarrow$ $\mathcal{P}(Y)$ is induced by a subsurface inclusion $X \rightarrow Y$.

An interesting intermediate example is the Hatcher-Thurston complex: observe that, unlike in the case of pants complexes, puncturing a surface induces an injective simplicial map between the corresponding HatcherThurston complexes. It seems however possible to prove that all injections $\mathcal{H} T(X) \rightarrow \mathcal{H} T(Y)$ are obtained as a combination of puncturing and subsurface inclusions.

3.2. Automorphisms and injective endomorphisms. In this section we discuss the following result, due to Ivanov [46] and McCarthy [71], and a few of its extensions:

Theorem 3.4 (Ivanov, McCarthy). Let $X$ be a surface of genus at least 3 . Then $\operatorname{Aut}(\operatorname{Map}(X))=\operatorname{Map}^{*}(X)$.

We now sketch the proof of Theorem 3.4 for closed surfaces $X$ of genus at least 4, using a simplified version of the arguments in [6]. The idea is to deduce from Theorem 2.7 that every automorphism

$$
\phi: \operatorname{Map}(X) \rightarrow \operatorname{Map}(X)
$$

induces an automorphism $\phi_{*}: \mathcal{C}_{n s}(X) \rightarrow \mathcal{C}_{n s}(X)$, which is in turn induced by a mapping class by a result of Irmak [43]. To define $\phi_{*}$ we proceed as follows. Given a non-separating curve $\gamma \subset X$ consider the Dehn twist $\delta_{\gamma}$ along $\gamma$. By Theorem 2.7, $\phi\left(\delta_{\gamma}\right)$ is a root of a multi-twist, which has infinite order since $\phi$ is injective. Let $\phi_{*}(\gamma)$ be the non-empty multicurve supporting any multi-twist power of $\phi\left(\delta_{\gamma}\right)$. The following lemma is the heart of the argument:

Lemma 3.5. If $\gamma \subset X$ is a non-separating curve then $\phi_{*}(\gamma)$ is also a nonseparating curve. Moreover, $\phi_{*}: \mathcal{C}_{n s}(X) \rightarrow \mathcal{C}_{n s}(X)$ is an automorphism. 
Proof. Note that the multicurve $\phi_{*}(\gamma)$ is $\phi\left(\delta_{\gamma}\right)$-invariant. First, we claim that each individual component of $\phi_{*}(\gamma)$ is preserved by $\phi\left(\delta_{\gamma}\right)$. Denoting by $X_{\gamma}$ the surface obtained from $X$ by removing the interior of a regular neighborhood of $\gamma$, recall that $\delta_{\gamma}$ is central in the image of the homomorphism $\iota_{\#}: \operatorname{Map}\left(X_{\gamma}\right) \rightarrow \operatorname{Map}(X)$ induced by the inclusion $X_{\gamma} \rightarrow X$. Therefore, $\phi\left(\iota_{\#}\left(\operatorname{Map}\left(X_{\gamma}\right)\right)\right.$ preserves the multicurve $\phi_{*}(\gamma)$.

Since $X_{\gamma}$ has genus at least 3 , a result of Paris [81] yields that every homomorphism from $\operatorname{Map}\left(X_{\gamma}\right)$ to a symmetric group on at most $4(g-1)+$ $4=4 g$ elements is trivial. Thus, noting that $\phi_{*}(\gamma)$ has at most $3 g-3$ components, we deduce that $\phi\left(\iota_{\#}\left(\operatorname{Map}\left(X_{\gamma}\right)\right)\right.$, and a fortiori also $\phi\left(\delta_{\gamma}\right)$, does not permute the components of $\phi_{*}(\gamma)$.

Next, we claim that $\phi_{*}(\gamma)$ is in fact a single curve. Arguing by contradiction, suppose that $\phi_{*}(\gamma)$ has $k \geq 2$ elements. As Dehn twists about non-separating curves are conjugate in $\operatorname{Map}(X)$, then $\phi_{*}\left(\gamma^{\prime}\right)$ also has $k$ elements for every $\gamma^{\prime} \subset X$ non-separating. Since $X$ is closed, we may extend $\gamma$ to a pants decomposition $P$ such that no two elements of $P$ together separate $X$. Then $\phi_{*}(P)$ consists of $(3 g-3) k$ possibly equal, but otherwise pairwise disjoint, curves; since $k \geq 2$, the pigeonhole principle implies that there are $\alpha, \beta \in P$ distinct such that $\phi_{*}(\alpha) \cap \phi_{*}(\beta) \neq \emptyset$. Now, $\operatorname{Map}(X)$ is generated by Dehn twists about a set of simple closed curves such that each one of them is disjoint from either $\alpha$ or $\beta$, and thus we deduce that $\phi(\operatorname{Map}(X))$ fixes the multicurve $\phi_{*}(\alpha) \cap \phi_{*}(\beta)$, contradicting that $\phi$ is an automorphism.

Therefore, $\phi_{*}(\gamma)$ is a single curve on $X$. We now prove that $\phi_{*}(\gamma)$ is non-separating. Indeed, the elements of $\phi_{*}(P)$ all have the same topological type, again because any two Dehn twists about non-separating curves are conjugate in $\operatorname{Map}(X)$, and there are at most $g-1$ separating curves on $X$ with the same topological type.

Having proved that $\phi_{*}(\gamma)$ is a non-separating curve for every such curve $\gamma \subset X$ we obtain that the rule $\gamma \rightarrow \phi_{*}(\gamma)$ defines a map $\phi_{*}: \mathcal{C}_{n s}(X) \rightarrow$ $\mathcal{C}_{n s}(X)$ of the non-separating curve complex of $X$. The self-map of $\mathcal{C}_{n s}(X)$ associated to $\phi^{-1}$ is the inverse of $\phi_{*}$, and hence the latter is an automorphism of $\mathcal{C}_{n s}(X)$.

Continuing with the proof of Theorem 3.4, note that it follows from Lemma 3.5 and from the rigidity of $\mathcal{C}_{n s}(X)$ [43] that there is a homeomorphism $f: X \rightarrow X$ such that $\phi\left(\delta_{\gamma}\right)$ is a root of a power of the Dehn twist along $f(\gamma)=\phi_{*}(\gamma)$. The $\phi$-equivariance of $\phi_{*}$ yields that $\phi\left(\delta_{\gamma}\right)$ fixes $\phi_{*}(\eta)$ for every non-separating curve $\eta \subset X$ disjoint from $\gamma$. This observation implies easily that $\phi\left(\delta_{\gamma}\right)=\delta_{f(\gamma)}$. In other words, the automorphisms $\phi$ and $g \mapsto f g f^{-1}$ agree on the set of Dehn twists along non-separating curves, and hence are identical because such Dehn twists generate $\operatorname{Map}(X)$. This concludes the discussion of Theorem 3.4. 
Theorem 3.4 remains true if one considers only finite index subgroups of mapping class groups. Indeed, Ivanov [45] proved that every automorphisms between finite index $\operatorname{subgroups}$ of $\operatorname{Map}(X)$ is the restriction of an automorphism of $\operatorname{Map}(X)$. As an immediate consequence, the abstract commensurator of the mapping class group is the extended mapping class group:

Corollary 3.6 (Ivanov). $\operatorname{Comm}(\operatorname{Map}(X))=\operatorname{Map}^{*}(X)$.

In the same spirit, combining results of Korkmaz [56], Irmak [41, 42], Bell-Margalit [12], Behrstock-Margalit [10] and Shackleton [86], one gets:

Theorem 3.7. Let $X$ be a surface other than $S_{0, n}$ for $n \leq 4, S_{1, n}$ for $n \leq 2$ or $S_{2,0}$. Let $\Gamma$ be a subgroup of finite index in $\operatorname{Map}(X)$. Then every injective homomorphism $\Gamma \rightarrow \operatorname{Map}(X)$ is the restriction of an automorphism of $\operatorname{Map}(X)$.

Recall that a group $G$ is co-Hopfian if every injective homomorphism $G \rightarrow G$ is an isomorphism. As a consequence of Theorem 3.7, we obtain:

Corollary 3.8. Let $X$ be a surface other than $S_{0, n}$ for $n \leq 4$, or $S_{1, n}$ for $n \leq 2$. Then every finite index subgroup of $\operatorname{Map}(X)$ is co-Hopfian.

The strategy of the proofs of the results we just mentioned is similar to the one of the proof of Theorem 3.4. One shows that the, say injective, homomorphism in question induces a simplicial automorphism of a rigid complex such as the curve complex. As was the case in the proof of Theorem 3.4 , the key idea to obtain this map is to exploit commutativity relations in $\operatorname{Map}(X)$. Note for instance that the subgroup of $\operatorname{Map}(X)$ generated by the Dehn twists along the components of a pants decomposition of $X$ is free abelian of maximal rank, by a result of Birman-Lubotzky-McCarthy [16].

3.3. General homomorphisms. We now discuss homomorphisms between different mapping class groups. On the one hand, the theme may be informally described as that "imposing certain topological conditions on the domain and target surfaces gives rise to strong restrictions on the homomorphisms that can appear". On the other, all known examples and results point towards an affirmative answer to the following vague question:

Question 3. Suppose that $X$ has genus at least 3. Does every homomorphism $\operatorname{Map}(X) \rightarrow \operatorname{Map}(Y)$ arise from a manipulation of surfaces?

While the phrase "manipulation of surfaces" is not precise, we hope that it will have acquired a more definite meaning by the end of this section.

The first result about homomorphisms between mapping class groups of distinct surfaces $X$ and $Y$ is a theorem of Ivanov-McCarthy [48], who proved that there are no injective homomorphisms when $\kappa(Y)=\kappa(X)+1$ :

Theorem 3.9 (Ivanov-McCarthy). If $X$ and $Y$ have empty boundary, $X$ has genus at least 3 and $\kappa(Y) \leq \kappa(X)+1$, then every injective homomorphism $\operatorname{Map}^{*}(X) \rightarrow \operatorname{Map}^{*}(Y)$ is induced by a homeomorphism $X \rightarrow Y$. 
In [48], Ivanov and McCarthy also consider some lower genus cases of Theorem 3.9; for $X$ and $Y$ both of genus zero, this theorem is due BellMargalit [12].

We remark that some relation between the complexities of the surfaces $X$ and $Y$ is necessary for Theorem 3.9 to hold. For instance, as explained in [48], one may obtain injective homomorphisms between mapping class groups of punctured surfaces as follows. Let $X$ be a surface with one puncture, and $\kappa: Y \rightarrow X$ a characteristic cover such that the peripheral loop on $X$ lifts. Since $\kappa$ is characteristic, every homeomorphism of $X$ lifts to a homeomorphism of $Y$; moreover, we can choose such lift to fix a preferred preimage of the puncture of $X$. In this way we obtain an injective homomorphism $\operatorname{Map}^{*}(X) \rightarrow \operatorname{Map}^{*}(Y)$ between the associated extended mapping class groups.

It is worth mentioning that, composing the homomorphism $\operatorname{Map}^{*}(X) \rightarrow$ $\operatorname{Map}^{*}(Y)$ just described with the homomorphism induced by forgetting all but one punctures of $Y$, one obtains an injective homomorphism mapping a pseudo-Anosov to a multi-twist [4]:

Theorem 3.10 (Aramayona-Leininger-Souto). Suppose $X$ has genus $g \geq 2$ and one puncture. Then there exist a surface $Y$ of genus $g^{\prime}>g$ and an injective homomorphism $\operatorname{Map}(X) \rightarrow \operatorname{Map}(Y)$ with the following property: there exists $f \in \operatorname{Map}(X)$ pseudo-Anosov such that $\phi(f)$ is a multi-twist.

Note that the construction above uses in a crucial way that $X$ has punctures. However, also in [4] we proved:

Theorem 3.11 (Aramayona-Leininger-Souto). For every closed surface $X$ of genus at least 2, there are a closed surface $Y \neq X$ and an injective homomorphism $\phi: \operatorname{Map}(X) \rightarrow \operatorname{Map}(Y)$.

The homomorphism needed to prove Theorem 3.11 is also constructed using covers, although this time the covers we employ are far from being characteristic.

Continuing with the discussion of Theorem 3.9, note that the assumption $\partial X=\emptyset$ is crucial: indeed, if $X$ is allowed to have boundary, there are numerous examples of injective homomorphisms $\operatorname{Map}(X) \rightarrow \operatorname{Map}(Y)$, with $\kappa(Y)=\kappa(X)+1$, as can be seen by considering the homomorphism induced by a subsurface embedding $X \rightarrow Y$.

Finally, observe that Theorem 3.9 does not hold for non-injective homomorphisms. Indeed, let $X$ be a closed surface and consider the homomorphism $\operatorname{Map}(X) \rightarrow \operatorname{Map}(Y)$ provided by Theorem 3.11. Puncturing $X$ often enough we obtain a surface $Z$ with $\kappa(Z)=\kappa(Y)$. Then the composition of $\operatorname{Map}(X) \rightarrow \operatorname{Map}(Y)$ with the homomorphism $\operatorname{Map}(Z) \rightarrow \operatorname{Map}(X)$ given by forgetting all the punctures of $Z$ is a non-injective, but also highly nontrivial, homomorphism that is clearly not induced by a homeomorphism between $Z$ and $Y$. 
This last example shows that in general there might be non-injective, but also non-trivial, homomorphisms, while there are no injective ones. In [6] we classified all non-trivial homomorphisms between mapping class groups of surfaces satisfying suitable genus bounds. Before stating this result, we need some terminology. Let $X$ and $Y$ be surfaces of finite topological type, consider the cusps as marked points, and denote the underlying (compact) surfaces by $|X|$ and $|Y|$. By an embedding $\iota: X \rightarrow Y$ we understand a continuous injective map $\iota_{\text {top }}:|X| \rightarrow|Y|$ with the property that whenever $y \in \iota_{\text {top }}(|X|) \subset|Y|$ is a marked point of $Y$ in the image of $\iota_{\text {top }}$, then $\iota_{\text {top }}^{-1}(y)$ is also a marked point of $X$. As remarked in [6], every embedding $\iota: X \rightarrow Y$ is isotopic to a composition of the three operations we have encountered earlier in this paper: filling punctures, deleting boundary components, and subsurface embeddings. Every embedding $\iota: X \rightarrow Y$ induces a (continuous) homomorphism Homeo $(X) \rightarrow \operatorname{Homeo}(Y)$ and hence a homomorphism

$$
\iota_{\#}: \operatorname{Map}(X) \rightarrow \operatorname{Map}(Y) .
$$

The content of the following theorem, proved in [6], is that subject to suitable genus bounds, every non-trivial homomorphism is in fact induced by an embedding:

Theorem 3.12 (Aramayona-Souto). Suppose that $X$ and $Y$ are surfaces of finite topological type, of genus $g \geq 6$ and $g^{\prime} \leq 2 g-1$ respectively; if $Y$ has genus $2 g-1$, suppose also that it is not closed. Then every nontrivial homomorphism

$$
\phi: \operatorname{Map}(X) \rightarrow \operatorname{Map}(Y)
$$

is induced by an embedding $X \rightarrow Y$.

Remark. Several special cases of Theorem 3.12 were obtained simultaneously and independently by Castel [24].

Note that the assumption in Theorem 3.12 that the genus of $Y$ be less than twice that of $X$ is necessary. Indeed, suppose that $X$ has non-empty connected boundary and let $Y$ be the double of $X$. Let $X_{1}, X_{2}$ be the two copies of $X$ inside $Y$, and for $x \in X$ denote by $x_{i}$ the corresponding point in $X_{i}$. Given a homeomorphism $f: X \rightarrow X$ fixing pointwise the boundary and the punctures define

$$
\hat{f}: Y \rightarrow Y, \quad \hat{f}\left(x_{i}\right)=(f(x))_{i} \forall x_{i} \in X_{i}
$$

The homomorphism

$$
\operatorname{Homeo}(X) \rightarrow \operatorname{Homeo}(Y), \quad f \mapsto \hat{f}
$$

induces a homomorphism $\phi: \operatorname{Map}(X) \rightarrow \operatorname{Map}(Y)$ that is not induced by an embedding $X \rightarrow Y$. Note that the Dehn twist about $\partial X$ is contained in the kernel of $\phi$, and thus $\phi$ descends to a (injective) homomorphism

$$
\operatorname{Map}\left(S_{g, 1}\right) \rightarrow \operatorname{Map}\left(S_{2 g}, 0\right)
$$


We now discuss a few particular cases of Theorem 3.12. First observe that there is no embedding $X \rightarrow Y$ if the genus of $Y$ is less than that of $X$; it follows that, under this assumption, every homomorphism $\operatorname{Map}(X) \rightarrow$ $\operatorname{Map}(Y)$ is trivial. This result is due, for $X$ closed, to Harvey-Korkmaz [38].

If $X$ is closed, then any embedding $X \rightarrow Y$ is necessarily a homeomorphism; as a consequence we deduce that if $X$ and $Y$ are non-homeomorphic closed surfaces of genus $g \geq 6$ and $g^{\prime} \leq 2 g-2$, respectively, then every homomorphism $\operatorname{Map}(X) \rightarrow \operatorname{Map}(Y)$ is trivial. This provides an affirmative answer to a conjecture of Berrick-Matthey, who proved in [13] that for every $m$ there are infinitely many values of $g$ for which every homomorphism $\operatorname{Map}\left(S_{g, 0}\right) \rightarrow \operatorname{Map}\left(S_{g+m, 0}\right)$ is trivial.

In addition, if $X$ has genus at least 6 (in fact 4, as explained in [6]) and empty boundary, then every non-trivial endomorphism $\operatorname{Map}(X) \rightarrow \operatorname{Map}(X)$ is induced by a self-homeomorphism of $X$; this generalizes Ivanov's and McCarthy's Theorem 3.4 to homomorphisms that are not necessarily injective.

See [6] for other corollaries of the above theorem.

The basic idea of the proof of Theorem 3.12 is similar to the proof of Theorem 3.4 sketched above: one derives from Theorem 2.7 that $\phi$ maps Dehn twists along non-separating curves to Dehn twists along non-separating curves, and hence induces a map $\phi_{*}: \mathcal{C}_{n s}(X) \rightarrow \mathcal{C}_{n s}(Y)$. Since $\phi_{*}$ may well fail to be injective, we cannot use directly any known rigidity result for the curve complex - recall in addition that, if the genus of $Y$ is larger than that of $X$, then there is a number of exotic embeddings $\mathcal{C}(X) \rightarrow \mathcal{C}(Y)$. We circumvent this problem by exploiting the presence of the homomorphism $\phi: \operatorname{Map}(X) \rightarrow \operatorname{Map}(Y)$ : although it is not made explicit in [6], the argument somehow boils down to a rigidity theorem for $\phi$-equivariant maps between curve complexes.

\section{LIE THEORETIC SUPERRIGIDITY}

Having discussed in the previous section results in the spirit of the folkloric take on superrigidity, we now explore a possible analogy with the Lie theoretic version of Margulis's theorem. Recall that the latter asserts that homomorphisms between higher rank lattices virtually arise from homomorphisms between the ambient Lie groups. The first difficulty one faces when even thinking of extending this result to the setting of mapping class groups is the lack of an ambient group, as we discuss next.

4.1. The Morita-Markovic theorems. By definition $\operatorname{Map}(X)$ is defined as a quotient of $\operatorname{Homeo}(X)$ :

$$
1 \rightarrow \operatorname{Homeo}_{0}(X) \rightarrow \operatorname{Homeo}(X) \rightarrow \operatorname{Map}(X) \rightarrow 1
$$

It is also well-known that one can replace the group $\operatorname{Homeo}(X)$ by many other groups. What we mean by this is just the observation that whenever $\mathcal{G}(X) \subset \operatorname{Homeo}(X)$ is a subgroup such that every mapping class has a 
representative in $\mathcal{G}(X)$, we have

$$
1 \rightarrow \mathcal{G}_{0}(X) \rightarrow \mathcal{G}(X) \rightarrow \operatorname{Map}(X) \rightarrow 1
$$

where $\mathcal{G}_{0}(X)=\mathcal{G}(X) \cap \mathrm{Homeo}_{0}(X)$. There are many interesting choices for such a group $\mathcal{G}(X)$, for instance the groups of diffeomorphisms, $C^{k_{-}}$ diffeomorphisms, analytic diffeomorphisms, etc... In all these cases it is an interesting problem to study if the sequence (4.1) splits. More generally, one can wonder if for a given subgroup $\Gamma$ of $\operatorname{Map}(X)$ there is a homomorphism $\Gamma \rightarrow \mathcal{G}(X)$ which makes the following diagram commute:

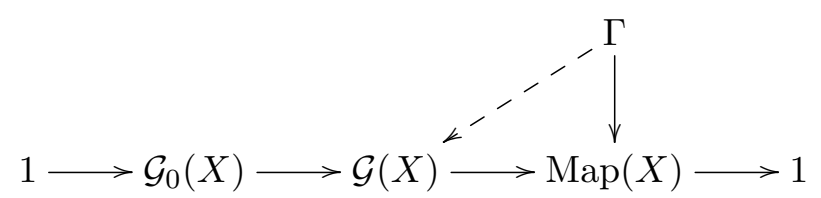

If the homomorphism $\Gamma \rightarrow \mathcal{G}(X)$ exists then we call it the lift, and say that $\Gamma$ lifts to $\mathcal{G}(X)$; otherwise, we say that $\Gamma$ does not lift. The first non-lifting result is due to Morita [75]:

Theorem 4.1 (Morita). If $X$ is a closed surface of genus $g \geq 3$, then $\operatorname{Map}(X)$ does not lift to the diffeomorphism group $\operatorname{Diff}(X)$ of $X$.

In [75], Morita proved Theorem 4.1 for $g \geq 5$ showing that the existence of a lift would imply the vanishing of certain cohomology classes of moduli space, which are known not to vanish. Later on, the genus bound was improved and the proofs became simpler. For instance, in [32] Franks-Handel derive the theorem above for $g \geq 3$ applying Thurston's stability theorem to fixed points of partially pseudo-Anosov elements. Also, while Morita's proof applies to lifting to the group of $C^{2}$-diffeomorphisms, the Franks-Handel argument applies to the group of $C^{1}$-diffeomorphisms as well. A much more involved argument shows that in fact the statement of Morita's theorem remains true for $g \geq 2$ and the group of homeomorphisms:

Theorem 4.2 (Markovic). If $X$ is a closed surface of genus $g \geq 2$, then $\operatorname{Map}(X)$ does not lift to $\operatorname{Homeo}(X)$.

Theorem 4.2 was proved by Markovic [64] for $g \geq 5$ and by Markovic-Saric [65] for $g \geq 2$.

So far, we have only considered the lifting problem for the whole mapping class group. In fact, Morita's original result for $g \geq 5$ applies also to finite index subgroups. At the other end of the spectrum, Kerckhoff proved [53] that finite subgroups of $\operatorname{Map}(X)$ lift to $\operatorname{Diff}(X)$. Also, it follows from Kerckhoff's result that virtually abelian and virtually free subgroups of $\operatorname{Map}(X)$ lift to $\operatorname{Diff}(X)$ as well. On the other hand, it has been conjectured that surface subgroups in $\operatorname{Map}(X)$ need not lift. The following result [14] points in this direction:

Theorem 4.3 (Bestvina-Church-Souto). If $X$ has genus at least 2 and $x \in$ $X$, then no finite index subgroup of $\pi_{1}(X, x) \subset \operatorname{Map}(X, x)$, the kernel of the 
Birman exact sequence

$$
1 \rightarrow \pi_{1}(X, x) \rightarrow \operatorname{Map}(X, x) \rightarrow \operatorname{Map}(X) \rightarrow 1
$$

lifts to the group of diffeomorphisms of $X$ fixing $x$.

In general, deciding which subgroups of $\operatorname{Map}(X)$ lift and which do not is a very interesting problem:

Question 4. Determine which subgroups of $\operatorname{Map}(X)$ lift to $\operatorname{Diff}(X)$. More concretely, determine for example if the subgroup generated by two Dehn twists along curves which intersect once lifts to $\operatorname{Diff}(X)$.

Until now, we have only considered the lifting problem as an algebraic problem, namely one about the (non-)existence of splittings of an exact sequence. There is however a different point of view, which we discuss next. Suppose for the sake of simplicity that $X$ is a closed surface. Every action $G \curvearrowright X$ of a group $G$ by orientation-preserving homeomorphisms induces a homomorphism $G \rightarrow \operatorname{Map}(X)$. From this point of view, Morita's (resp. Markovic's) theorem assert that there is no smooth (continuous) action of $\operatorname{Map}(X)$ on $X$ which induces the identity on $\operatorname{Map}(X)$. In [22], Cantat and Cerveau proved that in fact $\operatorname{Map}(X)$ does not act by analytic diffeomorphisms on any surface:

Theorem 4.4 (Cantat-Cerveau). Suppose that $X$ has genus at least 3 , that $Y$ is a closed surface with $\chi(Y)<0$, and let $\Gamma$ be a finite index subgroup of $\operatorname{Map}(X)$. There is no effective analytic action $\Gamma \curvearrowright Y$.

A word of warning: it would be perhaps natural to think that if there is no analytic or smooth action of a group on a manifold, then there should not be any continuous action either. In general, this is certainly not the case. For example, the extended mapping class group Map* $(X)$ of a closed surface $X$ acts on the unit tangent bundle $T^{1} X$ of $X$ in such a way that the composition

$$
\operatorname{Map}^{*}(X) \rightarrow \operatorname{Out}\left(\pi_{1}\left(T^{1} X\right)\right) \rightarrow \operatorname{Out}\left(\pi_{1}(X)\right) \simeq \operatorname{Map}^{*}(X)
$$

is the identity. In fact, the "natural" action is only Hölder, but it can be conjugated to a Lipschitz action. On the other hand, if $X$ has genus at least 12 , there is no such smooth action [89].

4.2. Inducing up homomorphisms. All the results we just discussed assert that $\operatorname{Map}(X)$ is not a subgroup of the "ambient groups" of homeomorphisms or diffeomorphisms of $X$. However, in all known examples, every homomorphism between mapping class groups of surfaces of sufficiently large genus is induced by a homomorphism between the corresponding "ambient groups". In fact, whenever any meaning is given to the sentence "manipulation of surfaces" as in Question 3, the following statement is true:

If a homomorphism $\phi: \operatorname{Map}(X) \rightarrow \operatorname{Map}(Y)$ arises from

a manipulation of surfaces, then there is a homomorphism 
$\Phi: \operatorname{Diff}_{c}(X) \rightarrow \operatorname{Diff}_{c}(Y)$ so that the following diagram commutes:

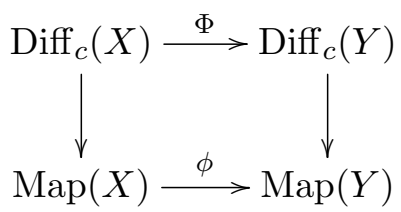

Here $\operatorname{Diff}_{c}(X)$ is the group of diffeomorphisms with compact support in $X \backslash \partial X$.

Continuing with the same notation, we say that $\Phi$ induces $\phi$.

Remark. Notice that if $X$ has cusps, then $\operatorname{Diff}_{c}(X) \cap \operatorname{Homeo}_{0}(X)$ is not the identity component of $\operatorname{Diff}_{c}(X)$. Therefore, but just for the sake of clarity, we assume for the rest of this section that $X$ is a compact surface, possibly with non-empty boundary.

The Lie theoretic version of Margulis Superrigidity asserts that homomorphisms between lattices (virtually) extend to homomorphisms of the ambient groups; in other words, homomorphisms between lattices are (virtually) induced by homomorphisms of the ambient groups. We wonder if the same is true for homomorphisms between mapping class groups:

Question 5. Suppose that $X$ has genus at least 3. Is it true that every homomorphism $\phi: \operatorname{Map}(X) \rightarrow \operatorname{Map}(Y)$ is (virtually) induced by a homomorphism $\Phi: \operatorname{Diff}_{c}(X) \rightarrow \operatorname{Diff}_{c}(Y)$ ?

In some way, question 5 is of motivational nature. At least the authors do not see any possibility of giving a positive answer, should that be the case, without previously having classified all homomorphisms between mapping class groups. We think however that understanding all homomorphisms $\operatorname{Diff}_{c}(X) \rightarrow \operatorname{Diff}_{c}(Y)$ would be interesting in its own right, and would also provide relevant information about the possible homomorphisms between mapping class groups. Any homomorphism $\operatorname{Diff}_{c}(X) \rightarrow \operatorname{Diff}_{c}(Y)$ should in fact arise from manipulations of surfaces... and it might be actually possible to prove that this is the case.

Question 6. Determine all non-trivial continuous homomorphisms $\Phi$ : $\operatorname{Diff}_{c}(X) \rightarrow \operatorname{Diff}_{c}(Y)$.

The remainder of this section is devoted to discuss a few known facts related to Question 6. To begin with, it is a classical theorem by Filipkiewicz [31] - valid in every dimension - that every isomorphism $\operatorname{Diff}_{c}(X) \simeq \operatorname{Diff}_{c}(Y)$ is induced by a diffeomorphism $X \backslash \partial X \simeq Y \backslash \partial Y$. Similar results are also known for isomorphisms between automorphisms groups of geometric structures such as, for instance, a volume form or a symplectic form $[8$, $9,84]$, but there are very few results on general homomorphisms between groups of diffeomorphisms. Only in dimension 1 is the situation completely understood: 
Theorem 4.5 (Mann). Suppose that $M$ and $N$ are 1-dimensional manifolds with empty boundary, and let $\Phi: \operatorname{Diff}_{c}(M) \rightarrow \operatorname{Diff}_{c}(N)$ be a homomorphism whose image $\Phi\left(\operatorname{Diff}_{c}(M)\right)$ acts transitively on $N$. Then $\Phi$ is induced by a diffeomorphism $M \simeq N$.

Remark. The formulation of Mann's theorem given here is not to be found in [61], but it is easily seen to be equivalent to the results therein.

The situation in dimension greater than 1 is much more complicated as indicated by the following result:

Theorem 4.6 (Aramayona-Leininger-Souto). Let $M$ be a closed connected manifold whose fundamental group surjects onto the symmetric group $\mathrm{Sym}_{3}$. Then there are a non-trivial connected finite cover $M^{\prime} \rightarrow M$ and an injective homomorphism $\operatorname{Diff}(M) \rightarrow \operatorname{Diff}\left(M^{\prime}\right)$ whose image acts transitively on $M^{\prime}$.

In [4], this theorem is only stated in dimension 2 , but the proof of the statement here is identical. Note that Theorem 3.11 follows directly from Theorem 4.6, and observe that the condition on the fundamental group seems to be of some importance:

Proposition 4.7. Suppose that $X=\mathbb{S}^{2}$ or that $X=\mathbb{T}^{2}$. If $Y$ is a surface and there is a non-trivial homomorphism $\Phi: \operatorname{Diff}_{0}(X) \rightarrow \operatorname{Diff}_{0}(Y)$ then $Y$ is diffeomorphic to $X$.

Proof. For the sake of concreteness we will only prove Proposition 4.7 if $X=\mathbb{S}^{2}$. Then $\mathrm{SO}_{3} \subset \operatorname{Diff}_{0}(X)$. Since Diff $0(X)$ is simple $[69,70]$, we obtain that either $\Phi$ is trivial or $\Phi\left(\mathrm{SO}_{3}\right)$ is isomorphic to $\mathrm{SO}_{3}$. In particular, the compact group $\mathrm{SO}_{3}$ acts on $Y$. This is only possible if $Y=\mathbb{S}^{2}$.

The simplicity of the identity component of the group of diffeomorphisms (with compact support) has numerous other consequences:

Theorem 4.8. Suppose that $X$ is a closed surface of genus $g \geq 6$ and that $Y$ has genus $2 \leq g^{\prime} \leq 2 g-2$. If $X \neq Y$, then there is no non-trivial homomorphism $\Phi: \operatorname{Diff}(X) \rightarrow \operatorname{Diff}(Y)$.

Proof. It follows from Theorem 3.12 that every homomorphism $\operatorname{Map}(X) \rightarrow$ $\operatorname{Map}(Y)$ is trivial. In particular, the image $\Phi(f)$ of any non-trivial $f \in$ $\operatorname{Diff}(X)$ of finite order has finite order and is isotopic to the identity. This implies that it is actually the identity and hence that $f \in \operatorname{Ker}(\Phi)$. Let now $g \in \operatorname{Diff}_{0}(X)$ be arbitrary without commuting with $f$, noting that $[f, g] \in$ $\operatorname{Ker}(\Phi) \cap \operatorname{Diff}_{0}(X)$. Since $\operatorname{Diff}_{0}(X)$ is simple, it follows that $\operatorname{Diff}_{0}(X) \subset$ $\operatorname{Ker}(X)$. Thus, the homomorphism $\Phi$ factors through a homomorphism $\Phi^{\prime}: \operatorname{Map}(X) \rightarrow \operatorname{Diff}(Y)$. Moreover, every finite order element of $\operatorname{Map}(X)$ belongs to the kernel of $\Phi^{\prime}$. Since $\operatorname{Map}(X)$ is generated by finite order elements, we have proved that $\Phi^{\prime}$, and hence $\Phi$, is trivial. 


\section{Geometric superrigidity}

As mentioned in section 1, in addition to the folkloric and Lie theoretic versions, Margulis Superrigidity also has a geometric interpretation in terms of maps between locally symmetric spaces. In this section, we explore to which extent there might be a geometric version of superrigidity in the context of mapping class groups. After reviewing some facts and known results about Teichmüller and moduli spaces, we discuss why the literal translation of Geometric Superrigidity cannot possibly hold. We propose instead a holomorphic version of superrigidity for homomorphisms between mapping class groups, motivated by rigidity results for maps between Kähler manifolds.

5.1. Background on Teichmüller and moduli spaces. We refer the reader to [39, 40, 78] for basic facts on Teichmüller space. Throughout this section we will assume that surfaces have finite analytic type; that is, they have finite topological type and empty boundary. Given such a surface $X$, the Teichmüller space $\mathcal{T}(X)$ is the space of (parabolic) holomorphic structures on $X$ up to isotopy fixing the punctures. Equivalently, $\mathcal{T}(X)$ is the space of isotopy classes of finite area complete hyperbolic metrics on $X$. The mapping class group $\operatorname{Map}(X)$ acts discretely on $\mathcal{T}(X)$ and it is due to Ahlfors [1] that $\mathcal{T}(X)$ admits a $\operatorname{Map}(X)$-invariant complex structure, with respect to which $\mathcal{T}(X)$ is biholomorphic to a bounded domain in $\mathbb{C}^{\kappa(X)}$. In particular, the moduli space

$$
\mathcal{M}(X)=\mathcal{T}(X) / \operatorname{Map}(X)
$$

is, by definition, a complex orbifold. Teichmüller space is a classifying space for proper actions $\underline{E}(\operatorname{Map}(X))$ of the mapping class group, meaning that the action is proper and that fixed-point sets of subgroups are either empty or contractible. In particular, there is a one-to-one correspondence between free homotopy classes of orbifold maps $\mathcal{M}(X) \rightarrow \mathcal{M}(Y)$ and conjugacy classes of homomorphisms $\operatorname{Map}(X) \rightarrow \operatorname{Map}(Y)$.

As mentioned in section 1 , the spaces $\mathcal{T}(X)$ and $\mathcal{M}(X)$ serve as the $\operatorname{Map}(X)$-analogs of the symmetric and locally symmetric space for a higher rank lattice. However, a first and major difference between Teichmüller space and a symmetric space is that, while a symmetric space has a huge group of isometries, Teichmüller space does not have many:

Theorem 5.1 (Avramidi [7]). Suppose that $X \neq S_{0,4}, S_{1,1}, S_{1,2}$ and let $\rho$ be a $\operatorname{Map}^{*}(X)$-invariant complete Finsler metric on $\mathcal{T}(X)$, of finite covolume. Then $\operatorname{Map}^{*}(X)$ is the full group of isometries of $(\mathcal{T}(X), \rho)$.

Avramidi's work builds on a previous result by Farb-Weinberger [30]. It does not apply to the Weil-Peterson metric because the latter is not complete, but in this case the result had already been established by MasurWolf [68], using again the rigidity of the curve complex (Theorem 3.1). 
5.2. Failure of geometric superrigidity. Avramidi's result may be interpreted as asserting that, unlike in the case of symmetric spaces, there is no preferred $\operatorname{Map}(X)$-invariant metric on Teichmüller space. Indeed, there is a number of well-known $\operatorname{Map}(X)$-invariant metrics on $\mathcal{T}(X)$ with respect to which $\mathcal{M}(X)$ has finite volume: these include the Teichmüller metric, the Weil-Petersson metric and McMullen's Kähler hyperbolic metric. See [58] for other examples as $\operatorname{Map}(X)$-invariant metrics on Teichmüller space.

The next result, which is a direct consequence of Theorem 3.10 above, states that the geometric version of Margulis Superrigidity has no literal translation to the context of mapping class groups:

Theorem 5.2 (Aramayona-Leininger-Souto). Suppose that $X=S_{g, 1}$ with $g \geq 2$. Then there are $Y \neq X$ and a map $\mathcal{M}(X) \rightarrow \mathcal{M}(Y)$ such that, when $\mathcal{M}(X)$ and $\mathcal{M}(Y)$ are endowed with any reasonable metric, is not homotopic to any totally geodesic map.

The reader might wonder what the term reasonable means in the statement of Theorem 5.2. For instance, it suffices that pseudo-Anosov elements have an axis (or quasi-axis) while multi-twists do not, so any of the metrics mentioned above is reasonable in this sense. The condition that the metric be reasonable stems from the fact that the map $\mathcal{M}(X) \rightarrow \mathcal{M}(Y)$ in Theorem 5.2 is proper and injective, and therefore one could first choose a metric on $\mathcal{M}(X)$, push it forward, and extend it to a metric on $\mathcal{M}(Y)$.

5.3. Holomorphic rigidity? Lacking a literal translation of the geometric version of Margulis Superrigidity, we now propose another potential version of superrigidity for maps between moduli spaces, which could be thought of as "holomorphic rigidity". We remark that the most naive possible interpretation of this, namely that every map $\mathcal{M}(X) \rightarrow \mathcal{M}(Y)$ be homotopic to a holomorphic map, cannot possibly hold either. Indeed, there is no holomorphic map in the homotopy class of maps $\mathcal{M}\left(S_{g, 1}\right) \rightarrow \mathcal{M}\left(S_{2 g, 0}\right)$ given by the homomorphism (3.1). However, there is an obvious holomorphic (and totally geodesic) map $\left.\mathcal{M}\left(S_{g, 1}\right)\right) \rightarrow \overline{\mathcal{M}}\left(S_{2 g, 0}\right)$ to the Mumford-Deligne compactification of $\mathcal{M}\left(S_{2 g, 0}\right)$. We recall that $\overline{\mathcal{M}}\left(S_{2 g, 0}\right)$ is a projective algebraic variety; as a topological space, it is the metric completion of $\mathcal{M}\left(S_{2 g, 0}\right)$ with respect to the Weil-Petersson metric [67].

The problem with the map $\mathcal{M}\left(S_{g, 1}\right) \rightarrow \mathcal{M}\left(S_{2 g, 0}\right)$ above is that the associated homomorphism $\operatorname{Map}\left(S_{g, 1}\right) \rightarrow \operatorname{Map}\left(S_{2 g, 0}\right)$ is not irreducible, meaning that its image fixes a curve on $Y$. On the other hand, every known example of an irreducible homomorphism between mapping class groups of analytically finite surfaces induces a holomorphic map between the corresponding moduli spaces. In fact, we think that the answer to the following question may well be positive:

Question 7. Suppose that $X$ and $Y$ have finite analytic type and suppose that $X$ has genus at least 3. Let $\phi: \operatorname{Map}(X) \rightarrow \operatorname{Map}(Y)$ be an irreducible homomorphism. Is there a $\phi$-equivariant holomorphic map $\mathcal{T}(X) \rightarrow \mathcal{T}(Y)$ ? 
Before sketching a possible approach to Question 7, we wish to point out a basic problem: even if one knew that the answer were positive, one would not gain much information about homomorphisms between mapping class groups, or equivalently about homotopy classes of maps between moduli spaces. The reason is that, while in the case of lattices Margulis Superrigidity implies that understanding homomorphisms between lattices boils down to problems in representation theory, and hence in linear algebra and combinatorics, not much is known about the possible holomorphic maps $\mathcal{M}(X) \rightarrow \mathcal{M}(Y)$. However, a relatively simple observation is that in every non-trivial homotopy class there is at most one holomorphic map [5]:

Theorem 5.3 (Aramayona-Souto). Suppose that $\Gamma \subset \operatorname{Map}(X)$ has finite index and let $\phi: \Gamma \rightarrow \operatorname{Map}(Y)$ be a homomorphism. Suppose also that $f_{1}, f_{2}: \mathcal{T}(X) \rightarrow \mathcal{T}(Y)$ are $\phi$-equivariant holomorphic maps. If $f_{1}$ is not constant, then $f_{1}=f_{2}$.

The idea of the proof of Theorem 5.3 is the following. The Weil-Petersson metric on moduli space is Kähler, geodesically convex, and has negative curvature. Eells and Sampson [27] derived from a variant of the Wirtinger inequality and Stokes' theorem, that every holomorphic map between Kähler manifolds with closed domain is harmonic. If, moreover, the target is negatively curved and geodesically convex, then finite energy harmonic maps are unique in their homotopy class. In particular, if the moduli space $\mathcal{M}(X)$ were closed, then Theorem 5.3 would follow directly from the Eells-Sampson theorem. Since moduli space is not closed, one shows that the boundary terms appearing when applying Stokes' theorem vanish, and that every holomorphic map $\mathcal{M}(X) \rightarrow \mathcal{M}(Y)$ has finite energy. To do so we endow the domain with McMullen's Kähler hyperbolic metric [73] instead of the WeilPeterson metric. The former metric has the virtue of being bi-Lipschitz to the Teichmüller metric, and thus, by the Kobayashi-hyperbolicity of the Teichmüller metric, the holomorphic maps $f_{i}$ are Lipschitz and hence have finite energy.

Note that Theorem 5.3 implies that every non-existence (resp. rigidity) theorem for homomorphisms between mapping class groups implies a nonexistence (rigidity) result for non-constant holomorphic maps between the corresponding moduli spaces. In this direction, combining Theorem 3.12 and Theorem 5.3 we obtain [5]:

Theorem 5.4 (Aramayona-Souto). Let $X$ and $Y$ be Riemann surfaces of finite analytic type of genus $g \geq 6$ and $g^{\prime} \leq 2 g-1$ respectively. Moreover, in the equality case suppose that $Y$ is not closed. Then, every non-constant holomorphic map $\mathcal{M}(X) \rightarrow \mathcal{M}(Y)$ is a forgetful map.

The result of Eells-Sampson on which we modeled the proof of Theorem 5.3 is perhaps the simplest instance of Kähler rigidity. A much more elaborate result along these lines is the following version of Siu's rigidity theorem [88] due to Carlson-Toledo [23]: 
Theorem 5.5 (Siu, Carlson-Toledo). Let $M$ be a closed Kähler manifold, and $N$ a hermitian locally symmetric space other than $\mathbb{H}^{2}$. Let $f: M \rightarrow N$ be a harmonic map, and suppose that there is a point $x \in M$ such that $d f_{x} T_{x} M=T_{f(x)} N$. Then $f$ is either holomorphic or antiholomorphic.

Recall that a symmetric space is hermitian if it is Kähler. We now give a very brief sketch of the proof of Theorem 5.5. First, one uses a Bochner formula, Stokes' theorem, and the fact that $N$ has non-positive hermitian curvature $R(X, Y, \bar{X}, \bar{Y}) \leq 0$ to prove $f$ is pluriharmonic; in other words, the restriction of $f$ to every germ of Riemann surface in $M$ is harmonic. Moreover, using that the curvature operator $R$ of $N$ is given in a very concrete fashion, it follows that if $d f_{x}$ is surjective then $f$ is either holomorphic or antiholomorphic at $x$. Analiticity, plus the existence of some $x$ where $d f_{x}$ is surjective, implies that $f$ is holomorphic on an open dense set, and hence holomorphic overall. This concludes the sketch of the proof of Theorem 5.5.

It should be noted that the condition that $d f_{x}$ is surjective at a point can be relaxed to a much weaker one, namely that the rank be sufficiently large. In fact, the strategy we just sketched has been implemented by Schumacher [87] for harmonic maps with respect to the Weil-Peterson metric:

Theorem 5.6 (Schumacher). Let $f: \overline{\mathcal{M}}(X) \rightarrow \overline{\mathcal{M}}(Y)$ be a harmonic map with $f(\mathcal{M}(X)) \subset \mathcal{M}(Y)$ and $f(\overline{\mathcal{M}}(X) \backslash \mathcal{M}(X)) \subset \overline{\mathcal{M}}(Y) \backslash \mathcal{M}(Y)$. If rank $d f \geq 4$ at a point, then $f$ is holomorphic or antiholomorphic.

In the light of Schumacher's theorem, proving that every irreducible homomorphism $\phi: \operatorname{Map}(X) \rightarrow \operatorname{Map}(Y)$ induces a harmonic map $\mathcal{M}(X) \rightarrow$ $\mathcal{M}(Y)$ should go a long way towards obtaining a positive answer to Question 7. Equivalently, one should prove that there is a $\phi$-equivariant harmonic map $\Phi: \mathcal{T}(X) \rightarrow \mathcal{T}(Y)$. As long as we endow $\mathcal{T}(X)$ with McMullen's Kähler metric and $\mathcal{T}(Y)$ with the Weil-Peterson metric, it follows from Theorem 2.7 that there is a $\phi$-equivariant map such that the induced map between moduli spaces has finite energy. Now, it follows from general principles that there is a harmonic map

$$
\Phi: \mathcal{T}(X) \rightarrow \overline{\mathcal{T}}(Y)
$$

where $\overline{\mathcal{T}}(Y)$ is the Weil-Peterson completion of $\mathcal{T}(Y)$. At this point, we face two difficulties:

(1) Prove that if $\phi$ is irreducible, then $\Phi(\mathcal{T}(X)) \subset \mathcal{T}(Y)$.

(2) Assuming (1), prove the analogous statement of Theorem 5.6.

We believe that (1) is the heart of the matter, but in any case, there is a number of technical problems one would need to surmount when trying to implement this strategy. Perhaps one could first try to prove:

Question 8. Suppose that $X$ has at least genus $3, \Gamma \subset \operatorname{Map}(X)$ be a finite index subgroup, and $N$ a closed manifold of constant negative curvature. Is it true that for every homomorphism $\phi: \Gamma \rightarrow \pi_{1}(N)$, and every $k \geq 3$, the map $H^{k}(\phi): H^{k}\left(\pi_{1}(N) ; \mathbb{R}\right) \rightarrow H^{k}(\Gamma ; \mathbb{R})$ is trivial? 
Question 8 is motivated by the following result due to Sampson [85]:

Theorem 5.7 (Sampson). Let $M$ be a closed Kähler manifold, $N$ a closed manifold of constant negative curvature, and $f: M \rightarrow N$ a harmonic map. Then $\operatorname{rank} d f \leq 2$.

We refer the reader to Toledo [94] for a beautiful survey on the topic of Kähler rigidity.

\section{REFERENCES}

[1] L. Ahlfors, The complex analytic structure of the space of closed Riemann surfaces, in Analytic Functions, Princeton Univ. Press (1960).

[2] J. Andersen, Mapping Class Groups do not have Kazhdan's Property (T), arXiv:0706.2184

[3] J. Aramayona, Simplicial embeddings between pants complexs, Geometriae Dedicata, 144, (2010).

[4] J. Aramayona, C. Leininger and J. Souto, Injections of mapping class groups, Geom. Topol. 13 (2009).

[5] J. Aramayona and J. Souto, Holomorphic maps between moduli spaces of Riemann surfaces, preprint.

[6] J. Aramayona and J. Souto, Homomorphisms between mapping class groups, to appear in Geom. Topol.

[7] G. Avramidi, Smith theory, $L^{2}$ cohomology, isometries of locally symmetric manifolds and moduli spaces of curves, arXiv:1106.1704.

[8] A. Banyaga, On isomorphic classical diffeomorphism groups I, Proc. AMS 98 (1986).

[9] A. Banyaga, On isomorphic classical diffeomorphism groups. II, J. Differential Geom. 28 (1988).

[10] J. Behrstock and D. Margalit, Curve complexes and finite index subgroups of mapping class groups, Geometriae Dedicata 118 (2006).

[11] B. Bekka, P. de la Harpe, and A. Valette, Kazhdan's property (T), New Mathematical Monographs, 11. Cambridge University Press, 2008.

[12] R. Bell and D. Margalit, Braid groups and the co-Hopfian property, Journal of Algebra $303(2006)$.

[13] A. J. Berrick and M. Matthey, Stable classical groups and strongly torsion generated groups. Comment. Math. Helv. 84 (2009).

[14] M. Bestvina, T. Church and J. Souto, Some groups of mapping classes not realized by diffeomorphisms, to appear in Comment. Math. Helv.

[15] M. Bestvina and K. Fujiwara, Bounded cohomology of subgroups of mapping class groups, Geom. Topol. 6 (2002).

[16] J. Birman, A. Lubotzky and J. McCarthy, Abelian and solvable subgroups of the mapping class groups, Duke Math. J. 50 (1983).

[17] J. S. Birman and C. Series, Geodesics with bounded intersection number on surfaces are sparsely distributed. Topology 24 (1985).

[18] A. Borel and J.-P. Serre, Corners and arithmetic groups, Comment. Math. Helv. 48 (1973).

[19] M. R. Bridson, Semi-simple actions of mapping class groups on CAT(0) spaces, in Geometry of Riemann surfaces, London Math. Soc. Lecture Note Ser. 368, 2010.

[20] M. Bridson and R. Wade, Actions of higher-rank lattices on free groups, Compos. Math. 147 (2011).

[21] M. Burger and N. Monod, Bounded cohomology of lattices in higher rank Lie groups, J. Eur. Math. Soc. 1 (1999). 
[22] S. Cantat and D. Cerveau, Analytic actions of mapping class groups on surfaces, J. Topol. 1 (2008).

[23] J. Carlson and D. Toledo, Harmonic mappings of Kähler manifolds to locally symmetric spaces, IHES Sci. Publ. Math. 69 (1989).

[24] F. Castel, Geometric representations of the braid groups, arXiv:1104.3698.

[25] F. Dahmani, V. Guirardel and D. Osin, Hyperbolic embeddings and rotating families in groups acting on hyperbolic spaces, arXiv:1111.7048.

[26] J. Dieudonné, On the automorphisms of the classical groups. With a supplement by Loo-Keng Hua, Mem. Amer. Math. Soc., 1951.

[27] J. Eells and J. H. Sampson, Harmonic mappings of Riemannian manifolds, Amer. J. Math. 86 (1964).

[28] B. Farb and D. Margalit, A primer on mapping class groups, Princeton Mathematical Series, 49. Princeton University Press, 2012.

[29] B. Farb and H. Masur, Superrigidity and mapping class groups, Topology 37 (1998).

[30] B. Farb and S. Weingerber, The intrinsic asymmetry and inhomogeneity of Teichmüller space, Duke Math. J, 155 (2010).

[31] R. Filipkiewicz, Isomorphisms between diffeomorphism groups, Ergodic Theory Dynamical Systems 2 (1982).

[32] J. Franks and M. Handel, Global fixed points for centralizers and Morita's theorem, Geom. Topol. 13 (2009).

[33] J. Franks and M. Handel, Triviality of some representations of $M C G\left(S_{g}\right)$ in $\operatorname{GL}(n, \mathbb{C}), \operatorname{Diff}\left(\mathbb{S}^{2}\right)$ and Homeo $\left(\mathbb{T}^{2}\right)$, to appear in Proc. AMS.

[34] H. Garland, A rigidity theorem for discrete subgroups, Trans. Amer. Math. Soc. 129 (1967).

[35] E. Grossman, On the residual finiteness of certain mapping class groups, J. London Math. Soc. (2) $9(1974 / 75)$.

[36] J. Harer, The virtual cohomological dimension of the mapping class group of an orientable surface, Invent. Math. 84 (1986).

[37] W. Harvey, Boundary structure of the modular group, in Riemann surfaces and related topics: Proceedings of the 1978 Stony Brook Conference Ann. of Math. Stud., 97, Princeton Univ. Press, 1981.

[38] W. Harvey and M. Korkmaz, Homomorphisms from mapping class groups, Bull. London Math. Soc. 37 (2005).

[39] J. H. Hubbard, Teichmüller theory and applications to geometry, topology, and dynamics. Matrix Editions, 2006.

[40] Y. Imayoshi and M. Taniguchi, An introduction to Teichmüller spaces. SpringerVerlag, Tokyo, 1992.

[41] E. Irmak, Superinjective simplicial maps of complexes of curves and injective homomorphisms of subgroups of mapping class groups, Topology 43 (2004).

[42] E. Irmak, Superinjective simplicial maps of complexes of curves and injective homomorphisms of subgroups of mapping class groups II, Topology and Its Applications 153 (2006).

[43] E. Irmak, Complexes of nonseparating curves and mapping class groups, Michigan Math. J. 54 (2006).

[44] E. Irmak and M. Korkmaz, Automorphisms of the Hatcher-Thurston complex, Israel Journal of Math 162 (2007).

[45] N. V. Ivanov, Automorphisms of complexes of curves and of Teichmüller spaces. Internat. Math. Res. Notices 14 (1997).

[46] N. Ivanov, Automorphisms of Teichmüller modular groups, in Topology and geometry - Rohlin Seminar, Lecture Notes in Math., 1346, Springer, 1988.

[47] N. Ivanov, Mapping class groups, in Handbook of geometric topology, North-Holland, 2002. 
[48] N.V. Ivanov and J. D. McCarthy, On injective homomorphisms between Teichmüller modular groups I, Invent. Math. 135 (1999).

[49] L. Ji, A tale of two groups: arithmetic groups and mapping class groups, in Handbook of Teichmüller theory. Volume III, IRMA Lect. Math. Theor. Phys., 17, Eur. Math. Soc., 2012.

[50] J. Jost, Nonpositive curvature: geometric and analytic aspects, Lectures in Mathematics ETH Zürich. Birkhäuser Verlag, 1997.

[51] J. Jost, Riemannian geometry and geometric analysis, Universitext. Springer, 2011.

[52] V. Kaimanovich and H. Masur, The Poisson boundary of the mapping class group, Invent. Math. 125 (1996).

[53] S. Kerckhoff, The Nielsen realization problem, Ann. of Math. 117 (1983).

[54] D. Kielak, Outer automorphism groups of free groups: linear and free representations, arXiv: 1202.0923.

[55] T. Koberda, Asymptotic linearity of the mapping class group and a homological version of the Nielsen-Thurston classification, Geom. Dedicata (2012).

[56] M. Korkmaz, Automorphisms of complexes of curves on punctured spheres and on punctured tori, Topology Appl. 95 (1999).

[57] M. Korkmaz, Low-dimensional homology groups of mapping class groups: a survey, Turkish J. Math. 26 (2002).

[58] K. Liu, X. Sun and S. T. Yau, Canonical metrics on the moduli space of Riemann surfaces I, J. Differential Geom. 68 (2004).

[59] E. Looijenga, Prym representations of mapping class groups, Geom. Dedicata 64 (1997).

[60] F. Luo, Automorphisms of the complex of curves. Topology 39 (2000).

[61] K. Mann, Homomorphisms between diffeomorphism groups, arXiv:1206.1196

[62] D. Margalit, Automorphisms of the pants complex, Duke Math. J. 121 (2004).

[63] G. Margulis, Discrete subgroups of semi-simple Lie groups, Ergebnisse der Mathematik und ihrer Grenzgebiete 17, Springer-Verlag, 1991.

[64] V. Markovic, Realization of the mapping class group by homeomorphisms, Invent. Math. 168 (2007).

[65] V. Markovic and D. Saric, The mapping class group cannot be realized by homeomorphisms, arXiv:0807.0182

[66] G. Masbaum, Quantum representations of mapping class groups, in Groupes et géométrie, 19-36, SMF Journ. Annu., 2003, Soc. Math. France, 2003.

[67] H. A. Masur, Extension of the Weil-Petersson metric to the boundary of Teichmüller space: Duke Mathematical Journal 43 (1976).

[68] H. Masur and M. Wolf, The Weil-Petersson isometry group, Geom. Dedicata 93 (2002).

[69] J. Mather, Commutators of diffeomorphisms Comment. Math. Helv. 49 (1974).

[70] J. Mather, Commutators of diffeomorphisms: II, Comment. Math. Helv. 50 (1975).

[71] J. D. McCarthy, Automorphisms of surface mapping class groups. A recent theorem of N. Ivanov, Invent. Math. 84 (1986).

[72] J. D. McCarthy and A. Papadopoulos, Simplicial actions of mapping class groups, in Handbook of Teichmüller theory. Volume III, IRMA Lect. Math. Theor. Phys., 17, Eur. Math. Soc., 2012.

[73] C. T. McMullen, The moduli space of Riemann surfaces is Kähler hyperbolic, Ann. of Math. 151 (2000).

[74] G. Mess, The Torelli groups for genus 2 and 3 surfaces, Topology 31 (1992).

[75] S. Morita, Characteristic classes of surface bundles, Invent. Math. 90 (1987).

[76] G. Mostow, Quasi-conformal mappings in n-space and the rigidity of the hyperbolic space forms, Publ. Math. IHES 34 (1968).

[77] G. Mostow, Strong rigidity of locally symmetric spaces, Annals of Mathematics Studies 78, Princeton University Press, 1973. 
[78] S. Nag, The complex analytic theory of Teichmüller spaces, John Wiley \& Sons, Inc., 1988.

[79] P. Pansu, Sous-groupes discrets des groupes de Lie: rigidité, arithméticité, Séminaire Bourbaki, Vol. 1993/94.

[80] A. Papadopoulos, Difféomorphismes pseudo-Anosov et automorphismes symplectiques de l'homologie, Ann. Sci. École Norm. Sup. (4) 15 (1982).

[81] L. Paris, Small index subgroups of the mapping class group, J. Group Theory 13 (2010).

[82] G. Prasad, Strong rigidity of $\mathbb{Q}$-rank 1 lattices, Invent. Math. 21 (1973).

[83] M. S. Raghunathan, On the first cohomology of discrete subgroups of semi-simple Lie groups, Amer. J. Math. 78 (1965).

[84] T. Rybicki, Isomorphisms between groups of diffeomorphisms, Proc. Amer. Math. Soc. 123 (1995).

[85] J. Sampson, Applications of harmonic maps to Kähler geometry, in Complex differential geometry and nonlinear differential equations, Contemp. Math. 49, AMS., 1986.

[86] K. J. Shackleton, Combinatorial rigidity in curve complexes and mapping class groups, Pacific Journal of Mathematics 230 (2007).

[87] G. Schumacher, Harmonic maps of the moduli space of compact Riemann surfaces. Math. Ann. 275 (1986).

[88] Y. Siu, The complex-analyticity of harmonic maps and the strong rigidity of compact Kähler manifolds, Ann. of Math. (2) 112 (1980).

[89] J. Souto, A remark on the action of the mapping class group on the unit tangent bundle, Ann. Fac. Sci. Toulouse Math. (6) 19 (2010).

[90] W. Thurston, The geometry and topology of 3-manifolds, Princeton lecture notes (1978-81).

[91] W. P. Thurston, On the geometry and dynamics of diffeomorphisms of surfaces, Bull. Amer. Math. Soc. 19 (1988).

[92] J. Tits, Buildings of spherical type and finite BN-pairs, Lecture Notes in Mathematics, Vol. 386, Springer, 1974.

[93] J. Tits, Travaux de Margulis sur les sous-groupes discrets de groupes de Lie, Séminaire Bourbaki, 28ème année (1975/76), Lecture Notes in Math., Vol. 567, Springer, 1977.

[94] D. Toledo, Rigidity theorems in Kähler geometry and fundamental groups of varieties, in Several complex variables, Math. Sci. Res. Inst. Publ., 37, Cambridge Univ. Press, 1999.

[95] A. Weil, On discrete subgroups of Lie groups I, Ann. Math. 72 (1960).

[96] A. Weil, On discrete subgroups of Lie groups II, Ann. Math. 75 (1962).

[97] D. Witte Morris, Introduction to Arithmetic Groups, math/0106063.

[98] R. J. Zimmer, Ergodic theory and semi-simple groups, Monogr. Math. 81, Birkhäuser, 1984.

School of Mathematics, Statistics and Applied Mathematics, National University of Ireland, Galway.

javier.aramayona@nuigalway.ie

Department of Mathematics,

University of British Columbia, Vancouver.

jsouto@math.ubc.ca 